\title{
Investigation on Magneto-thermoelastic Disturbances Induced by Thermal Shock in an Elastic Half Space Having Finite Conductivity under Dual Phase-lag Heat Conduction
}

\author{
Rakhi Tiwari*, Anil Kumar, Santwana Mukhopadhyay \\ Department of Mathematical Sciences, Indian Institute of Technology \\ (Banaras Hindu University), Varanasi 221 005, India \\ *E-mail: rakhibhu2117@gmail.com
}

Received: 20 April 2016; revised: 19 October 2016; accepted: 24 October 2016; published online: 16 November 2016

\begin{abstract}
The present work seeks to investigate the propagation of magneto-thermoelastic disturbances produced by a thermal shock in a finitely conducting elastic half-space in contact with vacuum. Normal load has been applied on the boundary of the existing media that is supposed to be permeated by a primary uniform magnetic field. We employ both the parabolic type (dual phase-lag magneto-thermoelasticity of type I (MTDPL-I)) and hyperbolic type (dual phase-lag magneto-thermoelasticity of type II (MTDPL-II)) dual phase-lag heat conduction models to account for the interactions among the magnetic, elastic and thermal fields. The integral transform technique is applied to solve the present problem and the analytical results of both cases have been obtained separately. A detailed analysis of results has been made in order to understand the nature of waves propagating inside the medium and the effects of the phase-lag parameters. The effect of the presence of magnetic field has been highlighted. Numerical results have also been obtained to analyze the effect of magnetic field on the behavior of the solution more clearly and a detailed analysis of the results predicted by two models has been presented. It has been noted that in some cases there are significant differences in the solution obtained in the contexts of MTDPL-I and MTDPL-II theory of magneto-thermoelasticity.
\end{abstract}

Key words: magneto-thermoelastic waves, finite conductivity, thermal shock, dual phase-lag heat conduction theory-I, dual phase-lag heat conduction theory-II

\section{INTRODUCTION}

The study of heat conduction theory has been made traditionally by using the classical Fourier law. This theory of heat conduction has been carried out successfully and it has been established that the results are in good agreement with experimental data for most of the analyzed experimental conditions [1,2]. But unfortunately, the approach of this Fourier law is not capable in giving accurate results in some situations, especially in situations involving very short times, high-heat-fluxes and very low temperatures [3-5]. Furthermore, it predicts heat propagation with infinite speed which is physically unrealistic. The classical coupled thermoelasticity theory developed by Biot [6] is based on Fourier law of the heat conduction and also suffers from this physical drawback that thermal signals propagate with infinite speed. Consequently, efforts have been made and different non Fourier models of heat conduction have been developed to surmount the limitations of the Fourier approach. The simplest and possibly the first one is suggested by Cattaneo [7] and also by Vernotte [8] by introducing the concept of thermal relaxation time parameter. Accordingly, some generalized theories have also been proposed with the aim of removing the paradox inherent in the classical coupled theory of thermoelasticity (CTE) given by Biot [6]. Firstly, Lord and Shulman [9] introduced the generalized thermoelasticity theory 
by employing the modified version of Fourier's law given by Cattaneo and Vernotte [7-8]. They introduced one thermal relaxation parameter in the governing equations of heat conduction equation which results in the hyperbolic type heat conduction equation. Later on, Green and Lindsay [10] proposed a more generalized theory of thermoelasticity in which Fourier law of heat conduction remained unchanged. Green and Naghdi [11] established a theory of thermoelasticity that permits the propagation of the thermal waves having finite speed. However, this theory does not accommodate the dissipation of energy of thermal signals. A more generalized version of the heat conduction theory for deformable bodies is also developed by Green and Naghdi [12] that accommodate the dissipation of energy of thermal waves.

It has been realized in recent years that the heat conduction theory of Catteneo and Vernotte also fails in some cases since this theory still establishes an instantaneous response between the temperature gradient and the energy transport. Hence, to surmount the drawbacks of the classical heat conduction model as well as the Cattaneo-Vernotte model, Tzou $[13,14]$ proposed the dual phase-lag (DPL) theory of heat conduction. This model establishes that either the temperature gradient may dominate the heat flux or that the heat flux may dominate the temperature gradient. This approach has been used to study the extensive variety of physical systems [15-18], structures with non linear boundary conditions [19], thermoelastic vibrations [17] etc. Two dual phase-lag thermoelastic models are subsequently developed by Chandrasekharaiah [20] by considering this dual phase-lag heat conduction model $[13,14]$. Several researchers have considered the dual phase-lag models to investigate the effects of employing the dual phase-lag heat conduction. We particularly mention the work in [21-28] and references there-in. Nowadays, increasing attention is being dedicated towards magneto-thermo-elastic interactions in a solid due to its several applications in many areas like plasma physics, geophysics and related topics. In the case of nuclear field, the extremely high temperature and the magnetic field that originates inside nuclear reactors influence their design and operations [29]. This terminology is known as the theory of magneto-thermo-elasticity. Basically, it is a combination of two different disciplines: the theory of electromagnetism and the theory of thermoelasticity. What must be recalled here is the significant contributions in the theory of magnetothermoelasticity and the theory of extended thermodynamics by several reserachers like Jordan and Eringen [30, 31], Maugin [32], Radzikowska et al. [33], Maruszewski et al. [34], Liu [35], Casas-Vazquez et al. [36], Lebon and Jou [37], Jou and Casas-Vazquez [38] and Maruszewski et al. [39]. Kaliski and Nowacki [40] investigated the magnetothermoelastic waves generated by a thermal shock in a perfectly conducting half-space in contact with vacuum. The coupling between temperature and strain fields was studied by Massalas and Dalamangas [41]. Then by using the thermal relaxation time of the Lord-Shulman theory [7], Roy- choudhuri and Chatterjee [42, 43] extended the problem [41] in the generalized thermoelasticity. Furthermore, by using the generalized theory of thermoelasticity developed by Lord and Shulman [7], Sharma and Dayal Chand [44] and Roychoudhuri and Banerjee (Mukhopadhyay) [45] investigated transient generalized magneto-thermoelastic waves in elastic half-spaces due to a normal load acting on the boundary of the half space.

In our work we employed the dual phase-lag thermoelasticity theory and studied a problem of elastic medium in the presence of the magnetic field with finite conductivity. The structure of the problem has been constructed in such a manner that both the normal load and thermal shock have been enforced on the boundary of the media due to which magneto-thermoelastic waves have been produced. We have made an attempt to find the solutions for the deformation, stress, temperature distributions and perturbed magnetic field by using two different theories: namely the 'dual phaselag heat conduction theory of magneto-thermoelasticity-I (MTDPL-I)' and the 'dual phase-lag heat conduction theory of magneto-thermoelasticity-II (MTDPL-II). Results obtained from both theories have been presented and discussed in a detailed way by highlighting the effects of phase-lag parameters due to the presence of the magnetic field.

\section{PROBLEM FORMULATION}

In our problem we have taken a homogeneous and isotropic finitely conducting elastic half space permeated by a primary uniform magnetic field such that a normal load and thermal shock have been applied on the boundary $x_{1}=0$, due to which magneto-thermoelastic disturbances have been initiated and allowed to propagate through the medium $x_{1} \geq 0$.

To formulate our problem we need to consider all the basic governing equations of magnetic, thermal and mechanical fields which are mutually interacting each other. Firstly, we consider the following equations given by Maxwell [46]:

$$
\begin{aligned}
\vec{\nabla} \times \vec{E} & =-\frac{\mu_{0}}{C} \cdot \frac{\partial \vec{h}}{\partial t}, \\
\vec{\nabla} \times \vec{h} & =\frac{4 \pi}{C} \vec{j}, \\
\vec{\nabla} \cdot \vec{h} & =0 .
\end{aligned}
$$

Generalized Ohm's law is given as

$$
\vec{j}=\lambda_{0}\left[\vec{E}+\frac{\mu_{0}}{C}\left(\dot{\vec{u}} \times \vec{H}_{0}\right)\right],
$$

where $\vec{E}, \vec{h}, \vec{H}_{0}$ represent the electric field, perturbed magnetic field and the initial constant magnetic field, respectively. $\mu_{0}$ and $\lambda_{0}$ are magnetic permeability and electrical conductivity of the medium, respectively and $C$ is the velocity of light. $\vec{j}$ is the current density vector and $\vec{u}$ is the 
displacement vector. Equation of motion including electromagnetic field is given by ([40])

$$
\begin{aligned}
& \mu \nabla^{2} \vec{u}+(\lambda+\mu) \vec{\nabla}(\vec{\nabla} \cdot \vec{u}) \\
+ & \frac{\mu_{0}}{4 \pi}\left[(\vec{\nabla} \times \vec{h}) \times \vec{H}_{0}\right]-\gamma \vec{\nabla} \theta=\rho \ddot{\vec{u}},
\end{aligned}
$$

where $\lambda, \mu$ are Lame constants, $\gamma=(3 \lambda+2 \mu) \alpha_{t}, \alpha_{t}$ being the coefficient of linear thermal expansion. $\theta=T-T_{0}$ where $T_{0}$ is the uniform reference temperature of the body, i.e. $\theta$ is the temperature above uniform reference temperature $T_{0}$. By employing dual phase-lag heat conduction model-II (DPL-II; (see Tzou [13] and Chandrashekharaiah [20])), we take the heat conduction equation for our study in the form

$$
\begin{aligned}
& K\left(1+\tau_{t} \frac{\partial}{\partial t}\right) \nabla^{2} \theta= \\
= & \left(1+\tau_{q} \frac{\partial}{\partial t}+\tau_{q}^{2} \frac{\partial^{2}}{\partial t^{2}}\right)\left(\rho C_{v} \dot{\theta}+\gamma T_{0} \dot{u}_{i, i}\right) .
\end{aligned}
$$

Special case: In the above equation, substituting $\tau_{q}^{2}=0$, we achieve the following heat conduction equation under dual phase-lag model-I (DPL-I)[[13],[20]]:

$$
K\left(1+\tau_{t} \frac{\partial}{\partial t}\right) \nabla^{2} \theta=\left(1+\tau_{q} \frac{\partial}{\partial t}\right)\left(\rho C_{v} \dot{\theta}+\gamma T_{0} \dot{u}_{i, i}\right)
$$

The above two heat conduction models are known as hyperbolic type dual phase-lag magneto-thermoelastic model-II (MTDPL-II) and parabolic type dual phase-lag magnetothermoelastic model-I (MTDPL-I), respectively, where $\tau_{q}$ represents phase-lag of heat flux vector and $\tau_{t}$ represents phase-lag of temperature gradient across the medium. Here 'Thomson effect' has been neglected due to its very small value. $C_{v}$ is the specific heat at constant strain and $\rho$ is the density. From equations (1)-(4), after elimination of $\vec{E}$ and $\vec{j}$, we achieve the following relation:

$$
\nabla^{2} \vec{h}-\beta \dot{\vec{h}}=-\beta \vec{\nabla} \times\left(\dot{\vec{u}} \times \vec{H}_{0}\right),
$$

where $\beta=\frac{4 \pi \lambda_{0} \mu_{0}}{C^{2}}$ For simplification, we assume that the magneto-thermoelastic waves propagated in the medium $x_{i} \geq 0$ depend on one direction i.e. $x_{1}$ and the time $t$. Furthermore, it has been assumed that the initial magnetic field vector is applicable towards $x_{3}$ axis i.e. $\vec{H}_{0}=$ $\left(0,0, H_{3}\right)$, where $H_{3}$ is a constant. Therefore, equations (1)(4) lead to

$$
\begin{gathered}
\vec{j}=\frac{C}{4 \pi}\left(0,-\frac{\partial h_{3}}{\partial x_{1}}, 0\right), \\
\dot{\vec{h}}=-\frac{C}{\mu_{0}}\left(0,0, \frac{\partial E_{2}}{\partial x_{1}}\right), \\
\vec{j}=\lambda_{0}\left[0,\left(E_{2}-\frac{\mu_{0} H_{3} u_{1}}{C}\right), 0\right] .
\end{gathered}
$$

Since wave is propagating in $x_{1}$ direction and we have assumed that the magnetic field has been applied in $x_{3}$ direction, then consequently electric field is given by $\vec{E}=$ $\left(0, E_{2}, 0\right)$, where

$$
E_{2}=-\frac{C}{4 \pi \lambda_{0}} \cdot \frac{\partial h_{3}}{\partial x_{1}}+\frac{\mu_{0} H_{3} \dot{u_{1}}}{C} .
$$

In view of the above assumptions, equations (5)-(8) reduce to the forms

$$
\begin{gathered}
(\lambda+2 \mu) \frac{\partial^{2} u_{1}}{\partial x_{1}^{2}}-\frac{\mu_{0} H_{3}}{4 \pi} \cdot \frac{\partial h_{3}}{\partial x_{1}}-\gamma \frac{\partial \theta}{\partial x_{1}}=\rho \cdot \frac{\partial^{2} u_{1}}{\partial t^{2}}, \\
K\left(1+\tau_{t} \frac{\partial}{\partial t}\right) \nabla^{2} \theta= \\
=\left(1+\tau_{q} \frac{\partial}{\partial t}+\tau_{q}^{2} \frac{\partial^{2}}{\partial t^{2}}\right)\left(\rho C_{v} \frac{\partial \theta}{\partial t}+\gamma T_{0} \frac{\partial^{2} u_{1}}{\partial x_{1} \partial t}\right), \\
K\left(1+\tau_{t} \frac{\partial}{\partial t}\right) \nabla^{2} \theta= \\
=\left(1+\tau_{q} \frac{\partial}{\partial t}\right)\left(\rho C_{v} \frac{\partial \theta}{\partial t}+\gamma T_{0} \frac{\partial^{2} u_{1}}{\partial x_{1} \partial t}\right), \\
\frac{\partial^{2} h_{3}}{\partial x_{1}^{2}}-\beta \frac{\partial h_{3}}{\partial t}=\beta H_{3} \frac{\partial^{2} u_{1}}{\partial x_{1} \partial t} .
\end{gathered}
$$

For simplicity, in what follows we will use the notations $u_{1}=u, x_{1}=x$.

Now, since the medium has been assumed to be in contact with vacuum, the above equations need to be added to the electrodynamic equations in vacuum. In vacuum, the system of equations of electrodynamics reduce to the following forms:

$$
\begin{gathered}
\left(\frac{\partial^{2}}{\partial x^{\prime 2}}-\frac{1}{C^{2}} \frac{\partial^{2}}{\partial t^{2}}\right) \tilde{h}_{3}=0, \\
\left(\frac{\partial^{2}}{\partial x^{\prime 2}}-\frac{1}{C^{2}} \frac{\partial^{2}}{\partial t^{2}}\right) \tilde{E}_{2}=0, \\
\dot{\overrightarrow{\tilde{h}}}=C\left(0,0, \frac{\partial \tilde{E}_{2}}{\partial x^{\prime}}\right) \\
\dot{\overrightarrow{\tilde{E}}}=C\left(0, \frac{\partial \tilde{h_{3}}}{\partial x^{\prime}}, 0\right)
\end{gathered}
$$

where $x^{\prime}=-x$.

For our present study, the equations (13), (14), (16), (17) constitute the system under MTDPL-II model and the equations (13), (15)-(17) constitute the system under MTDPL-I model. We will study both systems separately. 


\section{INITIAL AND BOUNDARY CONDITIONS}

In general, Maxwell's electro-magnetic stress tensor $T_{i j}$ in CGS unit is defined as $T_{i j}=\frac{\mu_{0}}{4 \pi}\left(h_{i} H_{j}+h_{j} H_{i}-\right.$ $\left.\delta_{i j} h_{k} H_{k}\right) \quad i, j, k=1,2,3$. Therefore, by using this relation we have taken for our problem,

$$
T_{11}=-\frac{\mu_{0} h_{3} H_{3}}{4 \pi}, \tilde{T}_{11}=-\frac{\tilde{h}_{3} H_{3}}{4 \pi},
$$

where $T_{11}$ and $\tilde{T}_{11}$ are the components of Maxwell's stress tensor in the elastic medium and in vacuum, respectively. The normal stress in the elastic medium is given by

$$
\sigma_{11}=(\lambda+2 \mu) \frac{\partial u}{\partial x}-\gamma \theta
$$

Therefore, we assume the following boundary conditions as

$$
\begin{gathered}
\sigma_{11}+T_{11}-\tilde{T}_{11}=\sigma_{0} H(t) \text { on } x=x^{\prime}=0, \\
E_{2}=\tilde{E}_{2}, \quad h_{3}=\tilde{h}_{3} \text { on } x=x^{\prime}=0,
\end{gathered}
$$

where $H(t)$ is the Heaviside unit function and $\sigma_{0}$ is a constant stress. The thermal boundary condition has been taken in the following manner:

$$
\theta(x, t)=\theta_{0} H(t) \quad \text { on } x=x^{\prime}=0,
$$

where $\theta_{0}$ is a constant. The initial conditions for the MTDPL-II model are assumed to be homogeneous and they are taken as

$$
\begin{gathered}
u(x, 0)=0, \quad \theta(x, 0)=0, \quad \frac{\partial u(x, 0)}{\partial t}=0, \\
\frac{\partial \theta(x, 0)}{\partial t}=0, \quad \frac{\partial^{2} u(x, 0)}{\partial t^{2}}=0, \quad \frac{\partial^{2} \theta(x, 0)}{\partial t^{2}}=0 .
\end{gathered}
$$

In the similar way, the initial conditions for MTDPL-I are considered as

$$
u(x, 0)=0, \quad \theta(x, 0)=0, \quad \frac{\partial u(x, 0)}{\partial t}=0, \quad \frac{\partial \theta(x, 0)}{\partial t}=0
$$

\section{SOLUTION OF THE PROBLEM}

In order to simplify the solution of the problem, we introduce the following notations and non dimensional quantities:

$$
\begin{aligned}
& \xi=\frac{C_{0} x}{k}, \\
& \tau=\frac{C_{0}^{2} t}{k}, \\
& u^{\prime}=\frac{C_{0}\left(\lambda+2 \mu+\alpha_{0}^{2} \rho\right) u}{k \gamma T_{0}}, \\
& z^{\prime}=\frac{\theta}{T_{0}}, k=\frac{K}{\rho C_{v}}, \\
& \epsilon=\frac{\gamma^{2} T_{0}}{C_{e}\left(\lambda+2 \mu+\alpha_{0}^{2} \rho\right)},
\end{aligned}
$$

$$
\begin{aligned}
C_{e} & =\rho C_{v}, \\
h_{3} & =h, \\
\eta_{1} & =\frac{\mu_{0} H_{3}}{4 \pi \gamma T_{0}}, \\
\eta_{2} & =\frac{1}{k \beta}, \\
\eta_{3} & =\frac{H_{3} \gamma T_{0}}{\rho C_{0}^{2}}, \\
\eta_{4} & =\frac{C_{0}^{2}}{4 \pi \lambda_{0} k}, \\
\eta_{5} & =\frac{\mu_{0} \gamma H_{3} T_{0}}{\rho C^{2}}, \\
C_{1}^{2} & =\frac{\lambda+2 \mu}{\rho}, \\
C_{0}^{2} & =C_{1}^{2}+\alpha_{0}^{2}, \\
\alpha & =\frac{C_{0}}{C}, \\
\alpha_{0}^{2} & =\frac{\mu_{0} H_{3}^{2}}{4 \pi \rho}, \\
\tau_{q}^{\prime} & =\frac{C_{0}^{2}}{k} \tau_{q}, \\
\tau_{t}^{\prime} & =\frac{C_{0}^{2}}{k} \tau_{t} .
\end{aligned}
$$

Using the above notations and non dimensional quantities on boundary conditions [(20)-(22)], we get the following simplified forms of our boundary conditions:

$$
\begin{gathered}
\frac{C_{1}^{2}}{C_{0}^{2}} u_{, \xi}^{\prime}-z^{\prime}+\eta^{\prime} \tilde{h}=\frac{\sigma_{0}}{\gamma T_{0}} H(\tau) \quad \text { on } \xi=\xi^{\prime}=0, \\
h=\tilde{h} \quad \text { on } \xi=\xi^{\prime}=0, \\
-\eta_{4} h_{, \xi \tau}+\eta_{5} u_{, \tau \tau}^{\prime}+\tilde{h}_{, \xi}=0 \quad \text { on } \xi=\xi^{\prime}=0, \\
z^{\prime}=\frac{\theta_{0}}{T_{0}} H(\tau) \quad \text { on } \xi=\xi^{\prime}=0,
\end{gathered}
$$

where we have used the notation $\eta^{\prime}=\frac{\left(1-\mu_{0}\right) H_{3}}{4 \pi \gamma T_{0}}$.

Further, the total stress $\sigma_{1}$ in the elastic half space is given by

$$
\sigma_{1}=\sigma_{11}+T_{11} .
$$

From the above equation, we get the dimensionless form of total stress as $\sigma^{\prime}$, where $\sigma^{\prime}=\frac{\sigma_{1}}{\gamma T_{0}}$.

Now, the initial conditions for MTDPL-II model become

$$
\begin{gathered}
u^{\prime}(\xi, 0)=0, \quad z^{\prime}(\xi, 0)=0, \quad \frac{\partial u^{\prime}}{\partial \tau}(\xi, 0)=0, \\
\frac{\partial z^{\prime}}{\partial \tau}(\xi, 0)=0, \quad \frac{\partial^{2} u^{\prime}}{\partial \tau^{2}}(\xi, 0)=0, \quad \frac{\partial^{2} z^{\prime}}{\partial \tau^{2}}(\xi, 0)=0 .
\end{gathered}
$$

Similarly, the initial conditions for MTDPL-I model are reduced to

$$
u^{\prime}(\xi, 0)=0, z^{\prime}(\xi, 0)=0, \frac{\partial u^{\prime}}{\partial \tau}(\xi, 0)=0, \frac{\partial z^{\prime}}{\partial \tau}(\xi, 0)=0 .
$$


Equation given by (17) get the form

$$
\tilde{h}_{, \xi \xi}-\alpha^{2} \tilde{h}_{, \tau \tau}=0, \quad \tilde{E}_{2, \xi^{\prime} \xi^{\prime}}-\alpha^{2} \tilde{E}_{2, \tau \tau}=0,
$$

where $\xi^{\prime}>0$ and $\xi^{\prime}=-\xi, \tilde{h_{3}}=\tilde{h}$. Now, we study the present problem in the contexts of two different models MTDPL-I and MTDPL-II separately.

\section{1. Case I: Magneto-Thermoelastic Dual Phase-lag Model-II (MTDPL-II)}

First, applying the above non dimensional quantities in equations (13),(14) and (16), we obtain the following equations:

$$
\begin{gathered}
\frac{C_{1}^{2}}{C_{0}^{2}} u_{, \xi \xi}^{\prime}-\eta_{1} h_{, \xi}-z_{, \xi}^{\prime}-u_{, \tau \tau}^{\prime}=0, \quad \xi>0, \\
\left(1+\tau_{t}^{\prime} \frac{\partial}{\partial t}\right) z_{, \xi \xi}^{\prime}-\left(1+\tau_{q}^{\prime} \frac{\partial}{\partial \tau}+\tau_{q}^{\prime 2} \frac{\partial^{2}}{\partial \tau^{2}}\right) \times \\
\left(z_{, \tau}^{\prime}+\epsilon u_{, \xi \tau}^{\prime}\right)=0, \quad \xi>0, \\
\eta_{2} h_{, \xi \xi}-h_{, \tau}=\eta_{3} u_{, \xi \tau}^{\prime}, \quad \xi>0 .
\end{gathered}
$$

On setting $\tau_{t}^{\prime}=0$ and $\tau_{q}^{\prime 2}=0$ in above equations (31)-(33), we find that the resulting equations are in agreement with the equations of [45]. The above equations given by (31)(33) constitute the set of coupled partial differential equations with coupled boundary conditions in three variables $u^{\prime}$, $z^{\prime}$ and $h$. It is very difficult to solve this system analytically, therefore for the purpose of simplification it is assumed that the perturbed magnetic field $h$ varies very slowly with distance so that $\frac{\partial^{2} h}{\partial \xi^{2}} \approx 0$.

Then, equations (33) and (31) reduce to

$$
\begin{gathered}
h=-\eta_{3} u_{, \xi}^{\prime}, \quad \xi>0, \\
u_{, \xi \xi}^{\prime}-z_{, \xi}^{\prime}-u_{, \tau \tau}^{\prime}=0, \quad \xi>0 .
\end{gathered}
$$

In order to solve the problem, we first apply Laplace transform to both sides of equation (35) and we obtain the following equation:

$$
\left(\frac{\partial^{2}}{\partial \xi^{2}}-s^{2}\right) \overline{u^{\prime}}-\frac{\partial \overline{z^{\prime}}}{\partial \xi}=0, \quad \xi>0
$$

where, the over-headed bars represent the fields in the Laplace transform domain. Equations (32) and (24)-(27) reduce to

$$
\begin{gathered}
\left(\left(1+\tau_{t}^{\prime} s^{2}\right) \frac{\partial^{2}}{\partial \xi^{2}}-\left(1+\tau_{q}^{\prime} s^{2}+\tau_{q}^{\prime 2} s^{3}\right) s\right) \overline{z^{\prime}-} \\
\epsilon s\left(1+\tau_{q}^{\prime} s^{2}+\tau_{q}^{\prime 2} s^{3}\right) \frac{\partial \overline{u^{\prime}}}{\partial \xi}=0, \quad \xi>0, \\
\frac{C_{1}^{2}}{C_{0}^{2}} \frac{\partial \bar{u}^{\prime}}{\partial \xi}-\overline{z^{\prime}}+\eta^{\prime} \overline{\tilde{h}}=\frac{\sigma_{0}}{\gamma T_{0}} \frac{1}{s} \quad \text { on } \xi=\xi^{\prime}=0, \\
\bar{h}=\overline{\tilde{h}} \quad \text { on } \xi=\xi^{\prime}=0,
\end{gathered}
$$

$$
\begin{gathered}
\eta_{5} s^{2} \bar{u}^{\prime}+\left(1-\eta_{4} s\right) \frac{\partial \bar{h}}{\partial \xi}=0 \quad \text { on } \xi=\xi^{\prime}=0, \\
\overline{z^{\prime}}=\frac{\theta_{0}}{T_{0}} \cdot \frac{1}{s} \quad \text { on } \xi=\xi^{\prime}=0 .
\end{gathered}
$$

Elimination of $\overline{u^{\prime}}$ from equation (36) and equation (37) yields

$$
\begin{aligned}
& {\left[\left(1+\tau_{t}^{\prime} s^{2}\right) \frac{\partial^{4}}{\partial \xi^{4}}-\left(s\left(1+\tau_{q}^{\prime} s^{2}+\tau_{q}^{\prime 2} s^{3}\right)+s^{2}\left(1+\tau_{t}^{\prime} s^{2}\right)+\right.\right.} \\
& \left.\left.\epsilon s\left(1+\tau_{q}^{\prime} s^{2}+\tau_{q}^{\prime 2} s^{3}\right)\right) \frac{\partial^{2}}{\partial \xi^{2}}+s^{3}\left(1+\tau_{q}^{\prime} s^{2}+\tau_{q}^{\prime 2} s^{3}\right)\right] \overline{z^{\prime}}=0 .
\end{aligned}
$$

The general solution of the above equation, vanishing at $\xi \rightarrow \infty$ is given by

$$
\bar{z}^{\prime}=A e^{-\lambda_{1} \xi}+B e^{-\lambda_{2} \xi},
$$

where $A$ and $B$ are arbitrary constants and $\lambda_{1}^{2}$ and $\lambda_{2}^{2}$ are the roots of the equation given below:

$$
\begin{aligned}
& {\left[\left(1+\tau_{t}^{\prime} s^{2}\right) \lambda^{4}-\left(s\left(1+\tau_{q}^{\prime} s^{2}+\tau_{q}^{\prime 2} s^{3}\right)+s^{2}\left(1+\tau_{t}^{\prime} s^{2}\right)\right.\right.} \\
& \left.\left.+\epsilon s\left(1+\tau_{q}^{\prime} s^{2}+\tau_{q}^{\prime 2} s^{3}\right)\right) \lambda^{2}+s^{3}\left(1+\tau_{q}^{\prime} s^{2}+\tau_{q}^{\prime 2} s^{3}\right)\right]=0 .
\end{aligned}
$$

$A, B$ are determined from equations (38),(41) and (43) as

$$
\begin{gathered}
A=\frac{\left(k_{1} \lambda_{2}^{2}\left(1+\tau_{t}^{\prime} s^{2}\right)-k_{2} s-k_{2} \tau_{q}^{\prime} s^{3}-k_{2} \tau_{q}^{\prime 2} s^{4}\right)}{s \eta^{\prime \prime}\left(\lambda_{2}^{2}-\lambda_{1}^{2}\right)\left(1+\tau_{t}^{\prime} s^{2}\right)}, \\
B=\frac{-\left(k_{1} \lambda_{1}^{2}\left(1+\tau_{t}^{\prime} s^{2}\right)-k_{2} s-k_{2} \tau_{q}^{\prime} s^{3}-k_{2} \tau_{q}^{\prime 2} s^{4}\right)}{s \eta^{\prime \prime}\left(\lambda_{2}^{2}-\lambda_{1}^{2}\right)\left(1+\tau_{t}^{\prime} s^{2}\right)},
\end{gathered}
$$

where, $k_{1}=\frac{\theta_{0} \eta^{\prime \prime}}{T_{0}}, k_{2}=\frac{\theta_{0} \eta^{\prime \prime}}{T_{0}}+\frac{\theta_{0} \epsilon}{T_{0}}+\frac{\sigma_{0} \epsilon}{\gamma T_{0}}$. By using equations (34), (37) and (43), we achieve the following analytical solution of displacement and perturbed magnetic field and total stress in the Laplace transform domain in terms of $A$ and $B$ as

$$
\begin{aligned}
& \overline{u^{\prime}}(\xi, s)=\frac{1}{\epsilon s\left(1+\tau_{q}^{\prime} s^{2}+\tau_{q}^{\prime 2} s^{3}\right)} \times \\
& {\left[A\left\{\frac{\left(1+\tau_{q}^{\prime} s^{2}+\tau_{q}^{\prime 2} s^{3}\right) s-\left(1+\tau_{t}^{\prime} s^{2}\right) \lambda_{1}^{2}}{\lambda_{1}}\right\} e^{-\lambda_{1} \xi}+\right.} \\
& \left.B\left\{\frac{\left(1+\tau_{q}^{\prime} s^{2}+\tau_{q}^{\prime 2} s^{3}\right) s-\left(1+\tau_{t}^{\prime} s^{2}\right) \lambda_{2}^{2}}{\lambda_{2}}\right\} e^{-\lambda_{2} \xi}\right], \\
& \bar{h}(\xi, s)= \\
& =-\frac{\eta_{3}}{\epsilon s\left(1+\tau_{q}^{\prime} s^{2}+\tau_{q}^{\prime 2} s^{3}\right)}\left[A \left\{-\left(1+\tau_{q}^{\prime} s^{2}+\tau_{q}^{\prime 2} s^{3}\right) s+\right.\right. \\
& \left.\left(1+\tau_{t}^{\prime} s^{2}\right) \lambda_{1}^{2}\right\} e^{-\lambda_{1} \xi}+B\left\{-\left(1+\tau_{q}^{\prime} s^{2}+\tau_{q}^{\prime 2} s^{3}\right) s+\right. \\
& \\
& \left.\left.\left(1+\tau_{t}^{\prime} s^{2}\right) \lambda_{2}^{2}\right\} e^{-\lambda_{2} \xi}\right],
\end{aligned}
$$




$$
\begin{aligned}
& \overline{\sigma^{\prime}}(\xi, s)= \\
& =\frac{1}{\epsilon s\left(1+\tau_{q}^{\prime} s^{2}+\tau_{q}^{\prime 2} s^{3}\right)}\left[A \left\{-\left(1+\tau_{q}^{\prime} s^{2}+\tau_{q}^{\prime 2} s^{3}\right) s+\right.\right. \\
& \left.\left(1+\tau_{t}^{\prime} s^{2}\right) \lambda_{1}^{2}\right\} e^{-\lambda_{1} \xi}+B\left\{-\left(1+\tau_{q}^{\prime} s^{2}+\tau_{q}^{\prime 2} s^{3}\right) s+\right. \\
& \left.\left.\left(1+\tau_{t}^{\prime} s^{2}\right) \lambda_{2}^{2}\right\} e^{-\lambda_{2} \xi}\right]-\left[A e^{-\lambda_{1} \xi}+B e^{-\lambda_{2} \xi}\right] .
\end{aligned}
$$

\section{2. Case II: Magneto-Thermoelastic Dual Phase-lag Model-I (MTDPL-I)}

The case when we apply $\tau_{q}^{\prime 2}=0$ in the above solutions of case I represent the case of MTDPL-I. Further, applying $\tau_{q}^{\prime 2}=0$ in equation (44), we get the following equation for magneto-thermoelastic dual phase-lag model-I (MTDPL-I):

$$
\begin{aligned}
& {\left[\left(1+\tau_{t}^{\prime} s^{2}\right) \lambda^{\prime 4}-\left(s\left(1+\tau_{q}^{\prime} s^{2}\right)+s^{2}\left(1+\tau_{t}^{\prime} s^{2}\right)+\right.\right.} \\
& \left.\left.\epsilon s\left(1+\tau_{q}^{\prime} s^{2}\right)\right) \lambda^{\prime 2}+s^{3}\left(1+\tau_{q}^{\prime} s^{2}\right)\right]=0 .
\end{aligned}
$$

We denote the roots of the above equation (47) as $\lambda_{1}^{\prime 2}$ and $\lambda_{2}^{\prime 2}$ so that the solutions in the context of MTDPL-I can be obtained from equations (43), (45), (46.1) and (46.2) by replacing $\lambda_{1}$ and $\lambda_{2}$ with $\lambda_{1}{ }^{\prime}, \lambda_{2}{ }^{\prime}$, respectively.

\section{SHORT-TIME APPROXIMATION}

It is clear from equations (44) and (47) that the roots of both equations are dependent on Laplace transform parameter $s$. The closed form analytical solutions of the above system in the physical domain is therefore a formidable task. However, phase-lag effects are short-lived; therefore, we attempt to understand the behavior of waves propagating through the medium by deriving the solutions applicable for very small values of time. Hence, in this section we concentrate our attention on small-time approximated analytical solutions for both the cases. For our analysis, we obtain our results for MTDPL-I and MTDPL-II theories separately.

\section{1. Case-I: Magneto-Thermoelastic Dual Phase-lag Model-II (MTDPL-II)}

Assuming $s$ to be very large, we obtain the solution of equation (44) for large $s$ as

$$
\begin{aligned}
\lambda_{1} & =\sqrt{\frac{a_{4}}{2 \tau_{t}^{\prime}}} s+\frac{a_{5}}{2 \sqrt{2 a_{4} \tau_{t}^{\prime}}}+\left(\frac{a_{4}{ }^{\frac{1}{2}}}{8 \sqrt{2 \tau_{t}^{\prime}}}\left(\frac{-a_{5}^{2}}{a_{4}^{2}}+\frac{4 a_{6}}{a_{4}}\right)-\right. \\
& \left.\frac{\sqrt{a_{4}}}{2 \sqrt{2 \tau_{t}^{\prime 3}}}\right) \frac{1}{s}+O\left(s^{-2}\right), \\
\lambda_{2} & =\sqrt{\frac{a_{7}}{2 \tau_{t}^{\prime}}} s+\frac{a_{8}}{2 \sqrt{2 a_{7} \tau_{t}^{\prime}}}+\left(\frac{a_{6} \frac{1}{2}}{8 \sqrt{2 \tau_{t}^{\prime}}}\left(\frac{-a_{7}^{2}}{a_{6}^{2}}+\frac{4 a_{8}}{a_{6}}\right)-\right. \\
& \left.\frac{\sqrt{a_{6}}}{2 \sqrt{2 \tau_{t}^{\prime 3}}}\right) \frac{1}{s}+O\left(s^{-2}\right),
\end{aligned}
$$

where $a_{1}=\left((1+8 \epsilon) \tau_{q}^{\prime 2}+\tau_{t}^{\prime}\right)^{2}-4 \tau_{q}^{\prime 2} \tau_{t}^{\prime}, a_{2}=2 \tau_{q}^{\prime 3}(1+$ $\epsilon)^{2}-2 \tau_{q}^{\prime} \tau_{t}^{\prime}$

$$
\begin{gathered}
a_{3}=(1+\epsilon)^{2} \tau_{q}^{\prime 2}+2 \tau_{t}^{\prime}+2 \tau_{q}^{\prime 2}(\epsilon-1), a_{4}=a_{1}^{\frac{1}{2}}+\tau_{t}^{\prime}+ \\
\tau_{q}^{\prime 2}(1+\epsilon), a_{5}=(1+\epsilon) \tau_{q}^{\prime}+\frac{a_{2}}{2 \sqrt{a_{1}}} ; \\
a_{6}=1+\frac{a_{1}^{\frac{1}{2}}}{8}\left(\frac{4 a_{3}}{a_{1}}-\frac{a_{2}^{2}}{a_{1}^{2}}\right), a_{7}=a_{1}^{\frac{1}{2}}+\tau_{t}^{\prime}-\tau_{q}^{\prime 2}(1+\epsilon),
\end{gathered}
$$

$a_{8}=(1+\epsilon) \tau_{q}^{\prime}-\frac{a_{2}}{2 \sqrt{a_{1}}}$. For the sake of convenience, we write $\lambda_{1}$ and $\lambda_{2}$ in the following form:

$$
\begin{aligned}
& \lambda_{1}=\frac{s}{v_{1}}+B_{1}+D_{1}\left(\frac{1}{s}\right)+O\left(s^{-2}\right), \\
& \lambda_{2}=\frac{s}{v_{2}}+B_{2}+D_{2}\left(\frac{1}{s}\right)+O\left(s^{-2}\right),
\end{aligned}
$$

where different notations in the above equations are given by

$$
\begin{aligned}
B_{1} & =\frac{a_{5}}{2 \sqrt{2 a_{4} \tau_{t}^{\prime}}}, \\
\frac{1}{v_{1}} & =\sqrt{\frac{a_{4}}{2 \tau_{t}^{\prime}}}, \\
D_{1} & =\left(\frac{a_{4}^{\frac{1}{2}}}{8 \sqrt{2 \tau_{t}^{\prime}}}\left(\frac{-a_{5}^{2}}{a_{4}^{2}}+\frac{4 a_{6}}{a_{4}}\right)-\frac{\sqrt{a_{4}}}{2 \sqrt{2 \tau_{t}^{\prime 3}}}\right), \\
B_{2} & =\frac{a_{8}}{2 \sqrt{2 a_{7} \tau_{t}^{\prime}}}, \\
\frac{1}{v_{2}} & =\sqrt{\frac{a_{7}}{2 \tau_{t}^{\prime}}}, \\
D_{2} & =\left(\frac{a_{6}^{\frac{1}{2}}}{8 \sqrt{2 \tau_{t}^{\prime}}}\left(\frac{-a_{7}^{2}}{a_{6}^{2}}+\frac{4 a_{8}}{a_{6}}\right)-\frac{\sqrt{a_{6}}}{2 \sqrt{2 \tau_{t}^{\prime 3}}}\right) .
\end{aligned}
$$

\section{1. 1. Solution in Laplace transform domain}

Substituting values of $A$ and $B$ and $\lambda_{1}$ and $\lambda_{2}$ in the expressions of temperature, displacement and perturbed magnetic field given by (equations (43),(45), and (46.1)), we achieve the solutions in terms of increasing powers of $\frac{1}{s}$ for MTDPL-II in the following forms:

$$
\begin{gathered}
\overline{z^{\prime}(\xi, s)=} \frac{v_{1}^{2} v_{2}^{2}}{\eta^{\prime \prime} \tau_{t}^{\prime}\left(v_{1}^{2}-v_{2}^{2}\right)}\left[\left(\frac{N_{1}}{s}+\frac{N_{2}}{s^{2}}\right) e^{-\left(B_{1}+\frac{s}{v_{1}}\right) \xi}-\right. \\
\left.\left(\frac{N_{1}^{\prime}}{s}+\frac{N_{2}^{\prime}}{s^{2}}\right) e^{-\left(B_{2}+\frac{s}{v_{2}}\right) \xi}\right],
\end{gathered}
$$

$$
\begin{aligned}
& \overline{u^{\prime}}(\xi, s)=\frac{v_{1}^{3} v_{2}^{2}}{\eta^{\prime \prime} \epsilon \tau_{q}^{\prime 2} \tau_{t}^{\prime}\left(v_{1}^{2}-v_{2}^{2}\right)}\left[M_{1} \frac{1}{s^{2}}+M_{2} \frac{1}{s^{3}}\right] e^{-\left(B_{1}+\frac{s}{v_{1}}\right) \xi}- \\
& \frac{v_{2}^{3} v_{1}^{2}}{\eta^{\prime \prime} \epsilon \tau_{q}^{\prime 2} \tau_{t}^{\prime}\left(v_{1}^{2}-v_{2}^{2}\right)}\left[M_{1}^{\prime} \frac{1}{s^{2}}+M_{2}^{\prime} \frac{1}{s^{3}}\right] e^{-\left(B_{2}+\frac{s}{v_{2}}\right) \xi},
\end{aligned}
$$$$
\bar{h}(\xi, s)=\frac{\eta_{3} v_{1}^{2} v_{2}^{2}}{\eta^{\prime \prime} \epsilon\left(v_{1}^{2}-v_{2}^{2}\right)}\left\{\left[Q_{1} \frac{1}{s}+Q_{2} \frac{1}{s^{2}}\right] e^{-\left(B_{1}+\frac{s}{v_{1}}\right) \xi}-\right.
$$$$
\left.\left[Q_{1}^{\prime} \frac{1}{s}+Q_{2}^{\prime} \frac{1}{s^{2}}\right] e^{-\left(B_{2}+\frac{s}{v_{2}}\right) \xi}\right\},
$$

where all constants used above are given by

$N_{1}=\frac{k_{1}}{v_{2}^{2}}+k_{1} \tau_{t}^{\prime} B_{2}^{2}-2 v_{1} v_{2} \frac{\left(v_{1} B_{2}-v_{2} B_{1}\right)}{\left(v_{1}^{2}-v_{2}^{2}\right)}\left(-k_{2} \tau_{q}^{\prime}+\frac{2 k_{1} B_{2} \tau_{t}^{\prime}}{v_{2}}\right)$, 


$$
\begin{gathered}
N_{2}=\left(\frac{2 k_{1} B_{2}}{v_{2}}-k_{2}\right)-2 v_{1} v_{2} \frac{\left(v_{1} B_{2}-v_{2} B_{1}\right)}{\left(v_{1}^{2}-v_{2}^{2}\right)}\left(\frac{k_{1}}{v_{2}^{2}}+k_{1} \tau_{t}^{\prime} B_{2}^{2}\right), \\
N_{1}^{\prime}=\frac{k_{1}}{v_{1}^{2}}+k_{1} \tau_{t}^{\prime} B_{1}^{2}-2 v_{1} v_{2} \frac{\left(v_{1} B_{2}-v_{2} B_{1}\right)}{\left(v_{1}^{2}-v_{2}^{2}\right)} \times \\
\left(-k_{2} \tau_{q}^{\prime}+\frac{2 k_{1} B_{1} \tau_{t}^{\prime}}{v_{1}}\right), \\
N_{2}^{\prime}=\left(\frac{2 k_{1} B_{1}}{v_{1}}-k_{2}\right)-2 v_{1} v_{2} \frac{\left(v_{1} B_{2}-v_{2} B_{1}\right)}{\left(v_{1}^{2}-v_{2}^{2}\right)}\left(\frac{k_{1}}{v_{1}^{2}}+k_{1} \tau_{t}^{\prime} B_{1}^{2}\right), \\
M_{1}=\left(\tau_{q}^{\prime 2}-\frac{\tau_{t}^{\prime}}{v_{1}^{2}}\right)\left(-k_{2} \tau_{q}^{\prime 2}+\frac{k_{1} \tau_{t}^{\prime}}{v_{2}^{2}}\right), \\
M_{2}=\left\{\left(B_{1} v_{1}\left(\tau_{q}^{\prime 2}-\frac{\tau_{t}^{\prime}}{v_{1}^{2}}\right)+\tau_{q}^{\prime}-2 B_{1} \tau_{t}^{\prime}\right)+\left(\tau_{q}^{\prime 2}-\right.\right. \\
\left.\frac{\tau_{t}^{\prime}}{v_{1}^{2}}\right)\left(\frac{2 v_{1} v_{2}\left(v_{1} B_{2}-v_{2} B_{1}\right)}{\left(v_{1}^{2}-v_{2}^{2}\right)}+\frac{1}{\tau_{q}^{\prime}}\right\}, \\
M_{1}^{\prime}=\left(\tau_{q}^{\prime 2}-\frac{\tau_{t}^{\prime}}{v_{2}^{2}}\right)\left(-k_{2} \tau_{q}^{\prime 2}+\frac{k_{1} \tau_{t}^{\prime}}{v_{1}^{2}}\right), \\
M_{2}^{\prime}=\left\{\left(B_{2} v_{2}\left(\tau_{q}^{\prime 2}-\frac{\tau_{t}^{\prime}}{v_{2}^{2}}\right)+\tau_{q}^{\prime}-2 B_{2} \tau_{t}^{\prime}\right)+\right. \\
\left(\tau_{q}^{\prime 2}-\frac{\tau_{t}^{\prime}}{v_{2}^{2}}\right)\left(\frac{2 v_{1} v_{2}\left(v_{1} B_{2}-v_{2} B_{1}\right)}{\left(v_{1}^{2}-v_{2}^{2}\right)}+\frac{1}{\tau_{q}^{\prime}}\right\}, \\
Q_{1}^{\prime}=\left(-k_{2} \tau_{q}^{\prime 2}+\frac{k_{1} \tau_{t}^{\prime}}{v_{1}}\right)\left(-\frac{1}{\tau_{t}^{\prime}}+\frac{1}{v_{1}^{2} \tau_{q}^{\prime 2}}\right), \\
\left.q_{1}^{\prime} k_{2}^{\prime 2}+\frac{k_{1} \tau_{t}^{\prime}}{v_{2}}\right)\left(-\frac{1}{\tau_{t}^{\prime}}+\frac{1}{v_{2}^{2} \tau_{q}^{\prime 2}}\right),
\end{gathered}
$$$$
Q_{2}=\left(-k_{2} \tau_{q}^{\prime 2}+\frac{k_{1} \tau_{t}^{\prime}}{v_{1}}\right)\left(-\frac{1}{\tau_{t}^{\prime}}+\frac{1}{v_{1}^{2} \tau_{q}^{\prime 2}}\right)+
$$$$
\left(-k_{2} \tau_{q}^{\prime 2}+\frac{k_{1} \tau_{t}^{\prime}}{v_{2}^{2}}\right)\left(\frac{2 B_{1}}{v_{1} \tau_{q}^{\prime 2}}-\frac{1}{\tau_{q}^{\prime} \tau_{t}^{\prime}}\right)-
$$$$
\left.\frac{2 v_{1} v_{2}\left(v_{1} B_{2}-v_{2} B_{1}\right)}{\left(v_{1}^{2}-v_{2}^{2}\right)}\right)\left(-k_{2} \tau_{q}^{\prime 2}+\frac{k_{1} \tau_{t}^{\prime}}{v_{1}}\right)\left(-\frac{1}{\tau_{t}^{\prime}}+\frac{1}{v_{1}^{2} \tau_{q}^{\prime 2}}\right)
$$$$
Q_{2}^{\prime}=\left(-k_{2} \tau_{q}^{\prime 2}+\frac{k_{1} \tau_{t}^{\prime}}{v_{2}}\right)\left(-\frac{1}{\tau_{t}^{\prime}}+\frac{1}{v_{2}^{2} \tau_{q}^{\prime 2}}\right)+
$$$$
\left(-k_{2} \tau_{q}^{\prime 2}+\frac{k_{1} \tau_{t}^{\prime}}{v_{1}^{2}}\right)\left(\frac{2 B_{2}}{v_{2} \tau_{q}^{\prime 2}}-\frac{1}{\tau_{q}^{\prime} \tau_{t}^{\prime}}\right)-
$$$$
\frac{2 v_{1} v_{2}\left(v_{1} B_{2}-v_{2} B_{1}\right)}{\left(v_{1}^{2}-v_{2}^{2}\right)}\left(-k_{2} \tau_{q}^{\prime 2}+\frac{k_{1} \tau_{t}^{\prime}}{v_{2}}\right)\left(-\frac{1}{\tau_{t}^{\prime}}+\frac{1}{v_{2}^{2} \tau_{q}^{\prime 2}}\right) .
$$

From equation (46.2), we further obtain the non dimensional total stress in the half space in Laplace transform domain as

$$
\begin{aligned}
& \bar{\sigma}^{\prime}(\xi, s)=\frac{v_{1}^{2} v_{2}^{2}}{\eta^{\prime \prime} \epsilon \tau_{q}^{\prime 2} \tau_{t}^{\prime}\left(v_{1}^{2}-v_{2}^{2}\right)}\left\{\left[P_{1} \frac{1}{s}+P_{2} \frac{1}{s^{2}}\right] e^{-\left(B_{1}+\frac{s}{v_{1}}\right) \xi}-\right. \\
& \left.\left[P_{1}^{\prime} \frac{1}{s}+P_{2}^{\prime} \frac{1}{s^{2}}\right] e^{-\left(B_{2}+\frac{s}{v_{2}}\right) \xi}\right\}-\frac{v_{1}^{2} v_{2}^{2}}{\eta^{\prime \prime} \tau_{t}^{\prime}\left(v_{1}^{2}-v_{2}^{2}\right)} \times \\
& {\left[\left(\frac{N_{1}}{s}+\frac{N_{2}}{s^{2}}\right) e^{-\left(B_{1}+\frac{s}{v_{1}}\right) \xi}-\left(\frac{N_{1}^{\prime}}{s}+\frac{N_{2}^{\prime}}{s^{2}}\right) e^{-\left(B_{2}+\frac{s}{v_{2}}\right) \xi}\right],}
\end{aligned}
$$

where

$$
\begin{gathered}
P_{1}=\left(-\tau_{q}^{\prime 2}+\frac{\tau_{t}^{\prime}}{v_{1}^{2}}\right)\left(-k_{2} \tau_{q}^{\prime 2}+\frac{k_{1} \tau_{t}^{\prime}}{v_{2}^{2}}\right), \\
P_{1}^{\prime}=\left(-\tau_{q}^{\prime 2}+\frac{\tau_{t}^{\prime}}{v_{2}^{2}}\right)\left(-k_{2} \tau_{q}^{\prime 2}+\frac{k_{1} \tau_{t}^{\prime}}{v_{1}^{2}}\right), \\
P_{2}=\left\{-\left(-\tau_{q}^{\prime 2}+\frac{\tau_{t}^{\prime}}{v_{1}^{2}}\right)\left(-k_{2} \tau_{q}^{\prime 2}+\frac{k_{1} \tau_{t}^{\prime}}{v_{2}^{2}}\right)\left(\frac{1}{\tau_{q}^{\prime}}+\right.\right. \\
\left.\frac{2 v_{1} v_{2}\left(v_{1} B_{2}-v_{2} B_{1}\right)}{\left(v_{1}^{2}-v_{2}^{2}\right)}\right)+\left(-\tau_{q}^{\prime 2}+\frac{\tau_{t}^{\prime}}{v_{1}^{2}}\right) \times \\
\left.\left(-k_{2} \tau_{q}^{\prime}+\frac{2 k_{1} B_{2} \tau_{t}^{\prime}}{v_{2}}\right)+\left(-\tau_{q}^{\prime 2}+\frac{\tau_{t}^{\prime}}{v_{1}^{2}}\right)\left(-k_{2} \tau_{q}^{\prime 2}+\frac{k_{1} \tau_{t}^{\prime}}{v_{2}^{2}}\right)\right\}, \\
P_{2}^{\prime}=\left\{-\left(-\tau_{q}^{\prime 2}+\frac{\tau_{t}^{\prime}}{v_{2}^{2}}\right)\left(-k_{2} \tau_{q}^{\prime 2}+\frac{k_{1} \tau_{t}^{\prime}}{v_{1}^{2}}\left(\frac{1}{\tau_{q}^{\prime}}+\right.\right.\right. \\
\left.\frac{2 v_{1} v_{2}\left(v_{1} B_{2}-v_{2} B_{1}\right)}{\left(v_{1}^{2}-v_{2}^{2}\right)}\right)+\left(-\tau_{q}^{\prime 2}+\frac{\tau_{t}^{\prime}}{v_{2}^{2}}\right) \times \\
\left.\left(-k_{2} \tau_{q}^{\prime}+\frac{2 k_{1} B_{1} \tau_{t}^{\prime}}{v_{1}}\right)+\left(-\tau_{q}^{\prime 2}+\frac{\tau_{t}^{\prime}}{v_{2}^{2}}\right)\left(-k_{2} \tau_{q}^{\prime 2}+\frac{k_{1} \tau_{t}^{\prime}}{v_{1}^{2}}\right)\right\} .
\end{gathered}
$$

This completes the solution in Laplace transform domain for the case of MTDPL-II model.

\section{1. 2. Solution in physical domain}

The solutions obtained in the previous section are given in Laplace transform domain. The solution of different fields in the physical domain can be derived by inverting the Laplace transforms involved in the expressions given by equations [(52)-(55)] for MTDPL-II case. By applying suitable formulae of Laplace inversion, we finally obtain the solution in physical domain for the case of MTDPL-II as follows:

$$
\begin{aligned}
& z^{\prime}(\xi, \tau)=\frac{v_{1}^{2} v_{2}^{2}}{\eta^{\prime \prime}\left(v_{1}^{2}-v_{2}^{2}\right)}\left[e ^ { - B _ { 1 } \xi } \left(N_{1} H\left(\tau-\frac{\xi}{v_{1}}\right)+\right.\right. \\
& \left.N_{2}\left(\tau-\frac{\xi}{v_{1}}\right) H\left(\tau-\frac{\xi}{v_{1}}\right)\right)-e^{-B_{2} \xi}\left(N_{1}^{\prime} H\left(\tau-\frac{\xi}{v_{2}}\right)+\right. \\
& \left.\left.N_{2}^{\prime}\left(\tau-\frac{\xi}{v_{2}}\right) H\left(\tau-\frac{\xi}{v_{2}}\right)\right)\right]
\end{aligned}
$$$$
u^{\prime}(\xi, \tau)=\frac{v_{1}^{3} v_{2}^{2}}{\eta^{\prime \prime} \epsilon \tau_{q}^{\prime 2}\left(v_{1}^{2}-v_{2}^{2}\right)} e^{-B_{1} \xi}\left[F_{1}\left(\tau-\frac{\xi}{v_{1}}\right) \times\right.
$$

$$
\begin{aligned}
& \left.H\left(\tau-\frac{\xi}{v_{1}}\right)+F_{2}\left(\tau-\frac{\xi}{v_{1}}\right)^{2} H\left(\tau-\frac{\xi}{v_{1}}\right)\right]- \\
& \frac{v_{1}^{3} v_{2}^{2}}{\eta^{\prime \prime} \epsilon \tau_{q}^{\prime 2}\left(v_{1}^{2}-v_{2}^{2}\right)} e^{-B_{2} \xi}\left[M_{1}^{\prime}\left(\tau-\frac{\xi}{v_{1}}\right) H\left(\tau-\frac{\xi}{v_{1}}\right)+\right. \\
& \left.M_{2}^{\prime}\left(\tau-\frac{\xi}{v_{1}}\right)^{2} H\left(\tau-\frac{\xi}{v_{1}}\right)\right],
\end{aligned}
$$




$$
\begin{aligned}
& h(\xi, \tau)=\frac{\eta_{3} v_{1}^{2} v_{2}^{2}}{\eta^{\prime \prime} \epsilon\left(v_{1}^{2}-v_{2}^{2}\right)}\left\{\left[Q_{1} H\left(\tau-\frac{\xi}{v_{1}}\right)+\right.\right. \\
& \left.Q_{2}\left(\tau-\frac{\xi}{v_{1}}\right) H\left(\tau-\frac{\xi}{v_{1}}\right)\right] e^{-B_{1} \xi}-\left[Q_{1}^{\prime} H\left(\tau-\frac{\xi}{v_{2}}\right)+(5\right. \\
& \left.\left.Q_{2}^{\prime}\left(\tau-\frac{\xi}{v_{2}}\right) H\left(\tau-\frac{\xi}{v_{2}}\right)\right] e^{-B_{2} \xi}\right\}, \\
& \sigma^{\prime}(\xi, \tau)=\frac{v_{1}^{2} v_{2}^{2}}{\eta^{\prime \prime} \epsilon \tau_{q}^{\prime 2} \tau_{t}^{\prime}\left(v_{1}^{2}-v_{2}^{2}\right)}\left\{\left[P_{1} H\left(\tau-\frac{\xi}{v_{1}}\right)+\right.\right. \\
& \left.\quad P_{2}\left(\tau-\frac{\xi}{v_{1}}\right) H\left(\tau-\frac{\xi}{v_{1}}\right)\right] e^{-B_{1} \xi}-\left[P_{1}^{\prime} H\left(\tau-\frac{\xi}{v_{2}}\right)+\right. \\
& \left.\left.\quad\left(\tau-\frac{\xi}{v_{2}}\right) H\left(\tau-\frac{\xi}{v_{2}}\right)\right] e^{-B_{2} \xi}\right\}-\frac{v_{1}^{2} v_{2}^{2}}{\eta^{\prime \prime} \tau_{t}^{\prime}\left(v_{1}^{2}-v_{2}^{2}\right)} \times \\
& \left\{\left[N_{1} H\left(\tau-\frac{\xi}{v_{1}}\right)+N_{2}\left(\tau-\frac{\xi}{v_{1}}\right) H\left(\tau-\frac{\xi}{v_{1}}\right)\right] e^{-B_{1} \xi}-\right. \\
& \left.\quad\left[N_{1}^{\prime} H\left(\tau-\frac{\xi}{v_{2}}\right)+N_{2}^{\prime}\left(\tau-\frac{\xi}{v_{2}}\right) H\left(\tau-\frac{\xi}{v_{2}}\right)\right] e^{-B_{2} \xi}\right\} .
\end{aligned}
$$

\section{2. Case-II: Magneto-Thermoelastic Dual Phase Lag Model-I (MTDPL-I)}

In a similar way like MTDPL-II, assuming $s$ to be very large, we obtain the solution of equation (47) for large $s$ as

$$
\begin{aligned}
& \lambda_{1}^{\prime}=C_{2}+s+\frac{B_{4}}{s}+O\left(s^{-2}\right), \\
& \lambda_{2}^{\prime}=\left(\frac{G_{1}}{\sqrt{s}}+\frac{\sqrt{s}}{c_{2}}\right)+O\left(s^{\frac{-3}{2}}\right),
\end{aligned}
$$

where the different constants which are independent of $s$ are given by

$$
\begin{aligned}
& B_{4}=16\left(2+\tau_{t}^{\prime}\right)+8 \tau_{q}^{\prime 2}\left(1+2(1+\epsilon)^{2}\right)-\frac{1}{2 \tau_{t}^{\prime}}-\frac{\epsilon^{2} \tau_{q}^{\prime 2}}{16 \tau_{t}^{\prime 2}}, \\
& C_{2}=\frac{\epsilon \tau_{q}^{\prime}}{4 \tau_{t^{\prime}}} \\
& G_{1}=\frac{B_{2} \tau_{q}^{\prime}}{2(2+\epsilon) \tau_{t}^{\prime}}, \\
& \frac{1}{c_{2}}=\sqrt{\frac{(2+\epsilon) \tau_{q}^{\prime}}{2 \tau_{t}^{\prime}}} .
\end{aligned}
$$

\section{2. 1. Solution in Laplace transform domain}

From the same pattern as in case V.1.1, applying $\tau_{q}^{\prime 2}=$ 0 and replacing $\lambda_{1}$ with $\lambda_{1}^{\prime}$ and $\lambda_{2}$ with $\lambda_{2}^{\prime}$ in equations [(43),(45) and (46.1)] and in the expressions of $A$ and $B$ we obtain the following results for MTDPL-I:

$$
\begin{aligned}
& \overline{z^{\prime}}(\xi, s)=\frac{1}{\eta^{\prime \prime} \tau_{t}^{\prime}\left(-1+\frac{2 G_{1}}{c_{2}}\right)}\left[\frac{S_{1}}{s^{2}}+\frac{S_{2}}{s^{3}}\right] e^{-\left(s+C_{2}\right) \xi}- \\
& \frac{1}{\eta^{\prime \prime} \tau_{t}^{\prime}\left(-1+\frac{2 G_{1}}{c_{2}}\right)}\left[\frac{S_{1}^{\prime}}{s}+\frac{S_{2}^{\prime}}{s^{2}}\right] e^{-\left(\frac{\sqrt{s}}{c_{2}}+\frac{G_{1}}{\sqrt{s}}\right) \xi}, \\
& \bar{u}^{\prime}(\xi, s)=\frac{1}{\eta^{\prime \prime} \tau_{q}^{\prime} \tau_{t}^{\prime} \epsilon}\left[\frac{L_{1}}{s^{2}}+\frac{L_{2}}{s^{3}}\right] e^{-\left(C_{2}+s\right) \xi}- \\
& \frac{c_{2}}{\eta^{\prime \prime} \tau_{q}^{\prime} \tau_{t}^{\prime} \epsilon}\left[\frac{L_{1}^{\prime}}{s^{\frac{3}{2}}}+\frac{L_{2}^{\prime}}{s^{\frac{5}{2}}}\right] e^{-\left(\frac{\sqrt{s}}{c_{2}}+\frac{G_{1}}{\sqrt{s}}\right) \xi},
\end{aligned}
$$

$$
\begin{aligned}
& \bar{h}(\xi, s)=\frac{1}{\eta^{\prime \prime} \tau_{q}^{\prime} \tau_{t}^{\prime} \epsilon\left(1-\frac{2 G_{1}}{c_{2}}\right)}\left[R_{1} \frac{1}{s}+R_{2} \frac{1}{s^{2}}\right] \times \\
& e^{-\left(C_{2}+s\right) \xi}-\frac{1}{\eta^{\prime \prime} \tau_{q}^{\prime} \tau_{t}^{\prime} \epsilon\left(1-\frac{2 G_{1}}{c_{2}}\right)} \times \\
& {\left[R_{1}^{\prime} \frac{1}{s}+R_{2}^{\prime} \frac{1}{s^{2}}\right] e^{-\left(\frac{\sqrt{s}}{c_{2}}+\frac{G_{1}}{\sqrt{s}}\right) \xi},}
\end{aligned}
$$

where different notations are given by

$$
\begin{aligned}
& S_{1}=\left(k_{1} \tau_{t}^{\prime}-\frac{k_{2} \tau_{q}^{\prime}}{c_{2}^{2}}\right), \\
& S_{2}=\left(\frac{-2 G_{1} k_{1} k_{2} \tau_{q}^{\prime} \tau_{t}^{\prime}}{c_{2}}-\frac{\left(-2 C_{2}+\frac{1}{c_{2}^{2}}\right)}{\left(-1+\frac{2 G_{1}}{c_{2}}\right)}\left(k_{1} \tau_{t}^{\prime}-\frac{k_{2} \tau_{q}^{\prime}}{c_{2}^{2}}\right)\right), \\
& S_{1}^{\prime}=k_{1} \tau_{t}^{\prime}, \\
& S_{2}^{\prime}=\left\{\left(-k_{2} \tau_{q}^{\prime}+2 C_{2} k_{1} \tau_{t}^{\prime}\right)+k_{1} \tau_{t}^{\prime} \frac{\left(-2 C_{2}+\frac{1}{c_{2}^{2}}\right)}{\left(-1+\frac{2 G_{1}}{c_{2}}\right)}\right\}, \\
& L_{1}=\tau_{t}^{\prime}\left(\frac{k_{1} \tau_{t}^{\prime}}{c_{2}^{2}}-k_{2} \tau_{q}^{\prime}\right), \\
& L_{2}==\frac{2 k_{1} \tau_{t}^{\prime} \tau_{q}^{\prime} G_{1}}{c_{2}}+\left(\frac{\tau_{t}^{\prime}}{c_{2}}-\tau_{q}^{\prime}-C_{2} \tau_{t}^{\prime}\right)\left(-k_{2} \tau_{q}^{\prime}+\frac{k_{1} \tau_{t}^{\prime}}{c_{2}^{2}}\right), \\
& L_{1}^{\prime}=\left(-\tau_{q}^{\prime}+\frac{\tau_{t}^{\prime}}{c_{2}^{2}}\right)\left(-k_{2} \tau_{q}^{\prime}+2 k_{1} C_{2} \tau_{t}^{\prime}\right), \\
& L_{2}^{\prime}=k_{1} \tau_{t}^{\prime}\left(2 C_{2}-\frac{1}{c_{2}}\right)\left(\tau_{q}^{\prime}-\frac{\tau_{t}^{\prime}}{c_{2}^{2}}\right)-c_{2} G_{1}\left(\tau_{q}^{\prime}-\frac{\tau_{t}^{\prime}}{c_{2}}\right), \\
& R_{1}=\tau_{t}^{\prime}\left(\frac{-k_{1} \tau_{t}^{\prime}}{c_{2}^{2}}-k_{2} \tau_{t}^{\prime}\right), \\
& R_{2}=\left[\frac{2 G_{1} k_{1}^{\prime} \tau_{t}^{2}}{c_{2}^{2}}+\left(2 C_{2} \tau_{t}^{\prime}-\tau_{q}^{\prime}\right)\left(-k_{2} \tau_{q}^{\prime}+\frac{k_{1} \tau_{t}^{\prime}}{c_{2}^{2}}\right)\right], \\
& R_{1}^{\prime}=k_{1} \tau_{t}^{\prime}\left(-\tau_{q}^{\prime}+\frac{\tau_{t}^{\prime}}{c_{2}^{2}}\right), \\
& R_{2}^{\prime}=\left[\frac{2 G_{1} k_{1} \tau_{t}^{\prime 2}}{+}\left(-\tau_{q}^{\prime}+\frac{\tau_{t}^{\prime}}{c_{2}^{2}}\right)\left(-k_{2} \tau_{q}^{\prime}+2 k_{1} C_{2} \tau_{t}^{\prime}\right)\right] .
\end{aligned}
$$

Similarly, we obtain the non dimensional total stress in Laplace transform domain (from eq. (46.2)) for MTDPL-I model is given by

$$
\begin{aligned}
& \overline{\sigma^{\prime}}(\xi, s)=\frac{1}{\eta^{\prime \prime} \in \tau_{q}^{\prime} \tau_{t}^{\prime}}\left[\left\{\frac{b_{1}}{s}+\frac{b_{1}^{\prime}}{s^{2}}\right\} e^{-\left(C_{2}+s\right) \xi}-\left\{\frac{d}{s}+\frac{d^{\prime}}{s^{2}}\right\} \times\right. \\
& \left.e^{-\left(\frac{G_{1}}{\sqrt{s}}+\frac{\sqrt{s}}{c_{2}}\right)}\right]-\frac{1}{\eta^{\prime \prime} \tau_{t}^{\prime}\left(-1+\frac{2 G_{1}}{c_{2}}\right)}\left[\frac{S_{1}}{s^{2}}+\right. \\
& \left.\frac{S_{2}}{s^{3}}\right] e^{-\left(s+C_{2}\right) \xi}+\frac{1}{\eta^{\prime \prime} \tau_{t}^{\prime}\left(-1+\frac{2 G_{1}}{c_{2}}\right)}\left[\frac{S_{1}^{\prime}}{s}+\frac{S_{2}^{\prime}}{s^{2}}\right] e^{-\left(\frac{\sqrt{s}}{c_{2}}+\frac{G_{1}}{\sqrt{s}}\right) \xi},
\end{aligned}
$$

where

$$
\begin{gathered}
b_{1}=2 C_{2} \tau_{t}^{\prime}\left(\frac{-2 G_{1}}{c_{2}}+\left(-2 C_{2}+\frac{1}{c_{2}}\right)\right)\left(-k_{2} \tau_{q}^{\prime}+\frac{1}{c_{2}^{2}}\right), \\
b_{1}^{\prime}=\left(\frac{-2 G_{1}}{c_{2}}+\left(-2 C_{2}+\frac{1}{c_{2}}\right)\right)\left(-k_{2} \tau_{q}^{\prime}+\frac{1}{c_{2}^{2}}\right) \times \\
\left(\frac{1}{\tau_{q}^{\prime}}\left(-k_{2} \tau_{q}^{\prime}+\frac{1}{c_{2}^{2}}\right)+\frac{2 G_{1}}{c_{2}}\left(-2 C_{2}+\frac{1}{c_{2}}\right)\right), \\
d=\left(2 C_{2}-\frac{1}{c_{2}}\right) k_{1}\left(1+\tau_{t}^{\prime}\right)+2 C_{2}-\frac{1}{c_{2}} \frac{\tau_{t}^{\prime}}{c_{2}^{2}} \times \\
\left(-k_{2} \tau_{q}^{\prime}+2 k_{1} C_{2} \tau_{t}^{\prime}\right)+\left(-k_{2} \tau_{q}^{\prime}+2 k_{1} C_{2} \tau_{t}^{\prime}\right)\left(\frac{2 G_{1} \tau_{t}^{\prime}}{c_{2}}-\tau_{q}^{\prime}\right),
\end{gathered}
$$




$$
\begin{gathered}
d^{\prime}=\left(\frac{1}{\tau_{q}^{\prime}}\left(-k_{2} \tau_{q}^{\prime}+2 k_{1} C_{2} \tau_{t}^{\prime}\right)+k_{1}\left(1+\tau_{t}^{\prime}\right)\left(-2 C_{2}+\frac{1}{c_{2}}\right)\right) \times \\
\left(\frac{\tau_{t}^{\prime}}{c_{2}^{2}}\right)+\left(\frac{2 G_{1} \tau_{t}^{\prime}}{c_{2}}-\tau_{q}^{\prime}\right)\left(2 C_{2}-\frac{1}{c_{2}^{2}}\right)\left(-k_{2} \tau_{q}^{\prime}+2 k_{1} C_{2} \tau_{t}^{\prime}\right) .
\end{gathered}
$$

This completes the solution in Laplace transform domain for the case of MTDPL-I model.

\section{2. 2. Solution in physical domain}

In a similar way like the case of MTDPL-II, the solution of different fields in physical domain for this case can be derived by inverting the Laplace transform involved in the expressions given by equations [(62)-(65)]. By using suitable formulae of Laplace inversion, we obtain the solution in physical domain for the present case as follows:

$$
\begin{aligned}
& z^{\prime}(\xi, \tau)=\frac{1}{\eta^{\prime \prime} \tau_{t}^{\prime}\left(-1+\frac{2 G_{1}}{c_{2}}\right)} \times \\
& e^{-C_{2} \xi}\left[S_{1}(\tau-\xi) H(\tau-\xi)+S_{2}(\tau-\xi)^{2} H(\tau-\xi)\right]- \\
& \frac{1}{\eta^{\prime \prime} \tau_{t}^{\prime}\left(-1+\frac{2 G_{1}}{c_{2}}\right)}\left[S_{1}^{\prime} \operatorname{Erfc}\left(\frac{\xi}{2 c_{2} \sqrt{\tau}}\right)+\right. \\
& \left.S_{2}^{\prime} 4 \tau i^{2} \operatorname{Erfc}\left(\frac{\xi}{2 c_{2} \sqrt{\tau}}\right)\right] \\
& u^{\prime}(\xi, \tau)=\frac{1}{\eta^{\prime \prime} \tau_{q}^{\prime} \tau_{t}^{\prime} \epsilon} e^{-C_{2} \xi}\left[L_{1}(\tau-\xi) H(\tau-\xi)+\right. \\
& \left.L_{2}(\tau-\xi)^{2} H(\tau-\xi)\right]-\frac{c_{2}}{\eta^{\prime \prime} \tau_{q}^{\prime} \tau_{t}^{\prime} \epsilon} \times \\
& {\left[F_{1}(4 \tau)^{\frac{1}{2}} i \operatorname{Erfc}\left(\frac{\xi}{2 c_{2} \sqrt{\tau}}\right)+\right.} \\
& \left.F_{2}(4 \tau)^{\frac{3}{2}} i^{3} \operatorname{Erfc}\left(\frac{\xi}{2 c_{2} \sqrt{\tau}}\right)\right], \\
& h(\xi, \tau)=\frac{1}{\eta^{\prime \prime} \tau_{q}^{\prime} \tau_{t}^{\prime} \epsilon\left(1-\frac{2 G_{1}}{c_{2}}\right)}\left[R_{1} H(\tau-\xi)+R_{2}(\tau-\xi) \times\right. \\
& H(\tau-\xi)] e^{-C_{2} \xi}-\frac{1}{\eta^{\prime \prime} \tau_{q}^{\prime} \tau_{t}^{\prime} \epsilon\left(1-\frac{2 G_{1}}{c_{2}}\right)}\left[R_{1}^{\prime} \operatorname{Erfc}\left(\frac{\xi}{2 c_{2} \sqrt{\tau}}\right)+\right. \\
& \left.R_{2}^{\prime} 4 \tau i^{2} \operatorname{Erfc}\left(\frac{\xi}{2 c_{2} \sqrt{\tau}}\right)\right] \\
& \sigma^{\prime}(\xi, \tau)=\frac{1}{\eta^{\prime \prime} \epsilon \tau_{q}^{\prime} \tau_{t}^{\prime}}\left[e ^ { - C _ { 2 } \xi } \left\{b_{1} H(\tau-\xi)+b_{1}^{\prime}(\tau-\xi) \times\right.\right. \\
& H(\tau-\xi)\}-\left\{d \operatorname{Erfc}\left(\frac{\xi}{2 c_{2} \sqrt{\tau}}\right)+\right. \\
& \left.\left.d^{\prime} 4 \tau i^{2} \operatorname{Erfc}\left(\frac{\xi}{2 c_{2} \sqrt{\tau}}\right)\right\}\right]-\frac{1}{\eta^{\prime \prime} \epsilon \tau_{t}^{\prime}\left(-1+\frac{2 G_{1}}{c_{2}}\right)} e^{-C_{2} \xi} \times \\
& {\left[S_{1}(\tau-\xi) H(\tau-\xi)+S_{2}(\tau-\xi)^{2} H(\tau-\xi)\right]+} \\
& \frac{1}{\eta^{\prime \prime} \epsilon \tau_{t}^{\prime}\left(-1+\frac{2 G_{1}}{c_{2}}\right)}\left[S_{1}^{\prime} \operatorname{Erfc}\left(\frac{\xi}{2 c_{2} \sqrt{\tau}}\right)+\right. \\
& \left.S_{2}^{\prime} 4 \tau i^{2} \operatorname{Erfc}\left(\frac{\xi}{2 c_{2} \sqrt{\tau}}\right)\right]
\end{aligned}
$$

where $i^{n} \operatorname{Erfc}(z)=\int_{z}^{\infty} i^{n-1} \operatorname{erf}(x) d x, i^{0} \operatorname{Erfc}(z)=$ $\operatorname{Erfc}(z), i^{-1} \operatorname{Erfc}(z)=\frac{2}{\sqrt{\pi}} e^{-z^{2}}$.

\section{ANALYSIS OF ANALYTICAL RESULTS}

The solution obtained in sections V.1.2 and V.2.2 for different fields in the physical domain indicate some significant information predicted by two different models of dual phaselags. From the short time approximated solutions given by equations (56)-(59) in the context of MTDPL-II model and the solutions given by (66)-(69) in the case of MTDPL-I we can observe that the solution of each field consists of two parts under both the theories of magneto-thermoelasticity (MTDPL-I and MTDPL-II); one part of solution is modified elastic and the other one is modified thermal in nature. In the case of the MTDPL-I model, the terms containing $H(\tau-\xi)$ represent modified elastic wave propagating with speed unity. Hence, in this case we conclude that the non dimensional speed of elastic wave is finite and equal to 1 , which imply that non dimensional speed of elastic wave is not effected by any of the phase-lag parameters $\tau_{q}^{\prime}$ and $\tau_{t}^{\prime}$ and it is also independent from the effect of magnetic field. We further note that the modified elastic wave in this case is propagating with an attenuation and $C_{2}$ is the attenuating coefficient of this wave. From the expression of $C_{2}$ it is evident that the attenuation coefficient is clearly dependent on phaselag parameters $\tau_{q}^{\prime}$ and $\tau_{t}^{\prime}$ in such a manner that on increasing $\tau_{q}^{\prime}$, attenuation coefficient increases and when we increase $\tau_{t}^{\prime}$, the value of attenuation coefficient decreases. Attenuation coefficient is also dependent on the magnetic field (see eq. (60)). It is observed that the value of attenuation coefficient decreases when magnetic field increases. The other part of solutions of each field in case of the MTDPL-I theory is not wave type, but a diffusive type and implies that the speed of thermal wave is not finite in this case. The solution is, however, influenced by the presence of the magnetic field.

The solution under the MTDPL-II model is completely different in nature. In this case, solution of each field like displacement, temperature, total stress consists of two different waves propagating with finite speeds and attenuating with distance. Here, $v_{1}$ and $v_{2}$ are the finite speeds of the modified elastic and modified thermal waves, respectively (since $v_{1}<v_{2}$ ). Accordingly, in the solutions of temperature, displacement and stress under MTDPL-II, the terms which contain $H\left(\tau-\frac{\xi}{v_{1}}\right)$, represent the contribution of elastic mode wave in the neighborhood of the wavefront $\xi=v_{1} \tau$; similarly the terms containing $H\left(\tau-\frac{\xi}{v_{2}}\right)$ represent the incorporation of thermal mode wave in the neighborhood of wavefront $\xi=v_{2} \tau$. The expressions for the speeds denote that both the speeds are influenced by the two phase-lags $\tau_{q}^{\prime}$ and $\tau_{t}^{\prime}$ and both are effected by the magnetic field, too. Furthermore, $B_{1}$ and $B_{2}$ represent attenuation coefficients for the modified elastic wave and modified thermal wave, respectively. $B_{1}$ and $B_{2}$ both are dependent on two phase-lags and 
also both are dependent on magneto-thermoelastic coupling constant $\epsilon$. This implies that the speed and attenuation of both the waves are influenced by the magnetic field under MTDPL-II model.

Furthermore, we observe in case of MTDPL-II that the physical fields such as temperature, stress and perturbed magnetic field have discontinuities with finite jumps at both the elastic and thermal wave fronts but displacement is continuous at both the wavefronts (see equations (56)-(59)).

However, in the context of the MTDPL-I model, we find different results. We observe here that only stress and perturbed magnetic field are discontinuous with finite jumps at elastic wavefront but temperature and displacement are free from any discontinuities (see equations (66)-(69)). This result is also in contrast with the results of [45] in which Lord Shulman model (LS model) has been used and in that model we see that temperature, stress and perturbed magnetic field suffer from discontinuities with finite jumps at both the elastic and thermal wavefronts and only displacement is free from any discontinuties. This indicates a distinct feature of dual-phase-lag model-I.

The finite jump discontinuities at the elastic wave front in MTDPL-I model and the finite jump discontinuities at elastic and thermal wave fronts in case of the MTDPL-II model for different fields are obtained as follows:

Finite jumps under the MTDPL-II model:

$$
\begin{aligned}
& {\left[z^{\prime+}-z^{\prime-}\right]_{\xi=v_{1} \tau}=\frac{v_{1}^{2} v_{2}^{2}}{\eta^{\prime \prime}\left(v_{1}^{2}-v_{2}^{2}\right)}\left[N_{1} e^{-B_{1} v_{1} \tau}\right],} \\
& {\left[z^{\prime+}-z^{\prime-}\right]_{\xi=v_{2} \tau}=\frac{-v_{1}^{2} v_{2}^{2}}{\eta^{\prime \prime}\left(v_{1}^{2}-v_{2}^{2}\right)}\left[N_{2} e^{-B_{2} v_{2} \tau}\right]} \\
& {\left[h^{+}-h^{-}\right]_{\xi=v_{1} \tau}=\frac{\eta_{3} v_{1}^{2} v_{2}^{2}}{\eta^{\prime \prime} \epsilon\left(v_{1}^{2}-v_{2}^{2}\right)}\left\{\left[Q_{1} e^{-B_{1} v_{1} \tau}\right],\right.} \\
& {\left[h^{+}-h^{-}\right]_{\xi=v_{2} \tau}=\frac{-\eta_{3} v_{1}^{2} v_{2}^{2}}{\eta^{\prime \prime} \epsilon\left(v_{1}^{2}-v_{2}^{2}\right)}\left\{\left[Q_{1}^{\prime} e^{-B_{2} v_{2} \tau}\right],\right.} \\
& {\left[\sigma^{+}-\sigma^{\prime-}\right]_{\xi=v_{1} \tau}=\frac{v_{1}^{2} v_{2}^{2}}{\eta^{\prime \prime} \epsilon \tau_{q}^{\prime 2} \tau_{t}^{\prime}\left(v_{1}^{2}-v_{2}^{2}\right)}\left[P_{1} e^{-B_{1} v_{1} \tau}\right]-} \\
& \frac{v_{1}^{2} v_{2}^{2}}{\eta^{\prime \prime} \tau_{t}^{\prime}\left(v_{1}^{2}-v_{2}^{2}\right)}\left\{\left[N_{1} e^{-B_{1} v_{1} \tau}\right]\right. \\
& {\left[\sigma^{+}-\sigma^{\prime}\right]_{\xi=v_{2} \tau}=-\left[\frac{v_{1}^{2} v_{2}^{2}}{\eta^{\prime \prime} \epsilon \tau_{q}^{\prime 2} \tau_{t}^{\prime}\left(v_{1}^{2}-v_{2}^{2}\right)}\left[P_{1}^{\prime} e^{-B_{2} v_{2} \tau}\right]-\right.} \\
& \left.\frac{v_{1}^{2} v_{2}^{2}}{\eta^{\prime \prime} \tau_{t}^{\prime}\left(v_{1}^{2}-v_{2}^{2}\right)}\left[N_{1}^{\prime} e^{-B_{2} v_{2} \tau}\right]\right]
\end{aligned}
$$

Finite jumps in the context of the MTDPL-I model:

$$
\begin{aligned}
{\left[\sigma^{+}-\sigma^{\prime}\right]_{\xi=\tau}=} & \frac{1}{\beta^{\prime \prime} \epsilon \tau_{q}^{\prime} \tau_{q}^{\prime}}\left[b_{1} e^{-C_{2} \tau}\right]- \\
& \frac{1}{\eta^{\prime \prime} \epsilon \tau_{t}^{\prime}\left(-1+\frac{2 G_{1}}{c_{2}}\right)}\left[S_{1} e^{-C_{2} \tau}\right], \\
{\left[h^{+}-h^{-}\right]_{\xi=\tau}=} & \frac{1}{\eta^{\prime \prime} \tau_{q}^{\prime} \tau_{t}^{\prime} \epsilon\left(1-\frac{2 G_{1}}{c_{2}}\right)}\left[R_{1} e^{-C_{2} \tau}\right] .
\end{aligned}
$$

\section{NUMERICAL RESULTS AND DISCUSSION}

In the previous section, we made an attempt to derive short-time approximated analytical results predicted by two models MTDPL-I and MTDPL-II that represent the effects of magneto-thermo-elastic interaction and highlighted the effects of phase-lag parameters and presence of magnetic field. However, in the present section we aim to illustrate the problem and instead of short-time approximated solutions we study the behaviour of numerical values of the physical fields like temperature, displacement and stress and perturbed magnetic field with distance under two different models: MTDPL-I and MTDPL-II. Here, we employ the numerical method proposed by Bellmen et al. [47] for the inversion of Laplace transforms, and compute the numerical values of these physical quantities by directly solving equations (43)-(47) numerically. We have used software Mathematica for our computational work. The results are plotted for MTDPL-I and MTDPL-II models separately to show the behaviour of the fields at three non-dimensional times 1.21, 0.69 and 0.13 . In order to observe the effects of magnetic field, we also plot the corresponding results under DPL-I and DPL-II models by assuming the magnetic parameters in our solution to be zero. We have chosen the copper material for our numerical computation. We have used the following physical data for the copper material: $k=1.14 \mathrm{~cm}^{2} / \mathrm{s}, T_{0}=20^{\circ} \mathrm{C}, H_{3}=10^{4}$ oersted; $\lambda=1.387 \times 10^{12} \mathrm{dyn} / \mathrm{cm}^{2}, \mu=0.448 \times 10^{12} \mathrm{dyn} / \mathrm{cm}^{2}$, $\rho=8.930 \mathrm{~g} / \mathrm{cm}^{3} ; \mu_{0}=1, \alpha_{t}=\left(16.5 \times 10^{-6}\right)^{o} \mathrm{C}^{-1}$ We further assume the following dimensionless values $\sigma_{0}^{\prime}=\frac{\sigma_{0}}{\gamma T_{0}}=1, \theta_{0}^{\prime}=\frac{\theta_{0}}{T_{0}}=1, \tau_{q}^{\prime}=0.2, \tau_{t}^{\prime}=0.15$.

\section{BEHAVIOUR OF TEMPERATURE UNDER MTDPL-I AND MTDPL-II}

It is clear from Figs 1, 2 and 3 that the results of temperature field under DPL-I and MTDPL-I are almost the same. Similarly, the results of DPL-II and MTDPL-II are also in complete agreement. However, the results under DPL-I and MDPL-I are significantly different from the results under DPL-II and MTDPL-II models. This implies that although the effect of magnetic field is not prominent in the behaviour of temperature field under MTDPL-I and MTDPL-II models, there are significant differences among the results of two different theories (MTDPL-I and MTDPL-II). This difference decreases as the time decreases. Although, the behaviour of temperature is in decreasing trend in all figures 


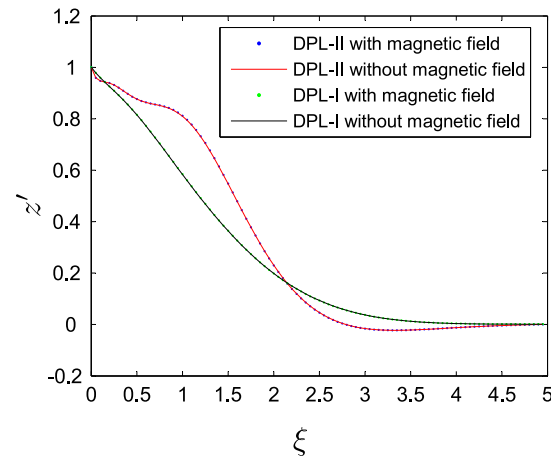

Fig. 1. Variation of temperature $z^{\prime}$ with distance $\xi$, time $=1.21$

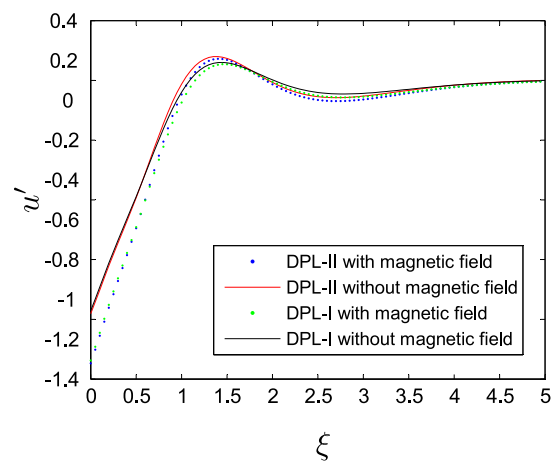

Fig. 4. Variation of displacement $u^{\prime}$ with distance $\xi$, time $=1.21$

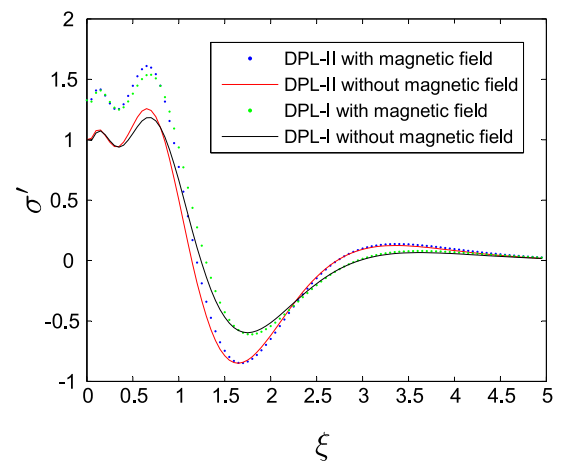

Fig. 7. Variation of stress $\sigma^{\prime}$ with distance $\xi$ time $=1.21$

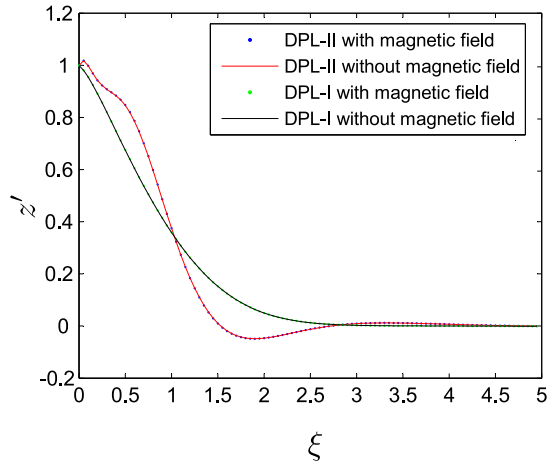

Fig. 2. Variation of temperature $z^{\prime}$ with distance $\xi$, time $=0.69$

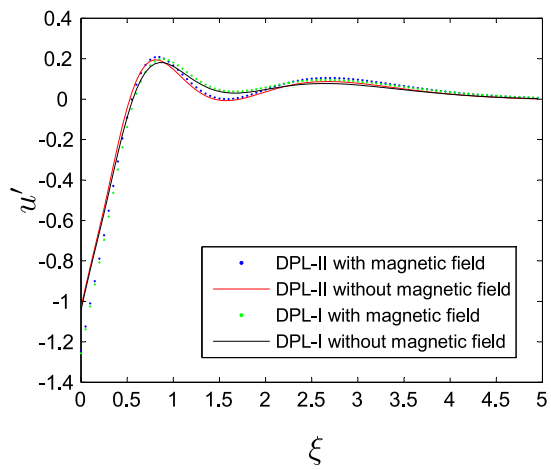

Fig. 5. Variation of displacement $u^{\prime}$ with distance $\xi$, time $=0.69$

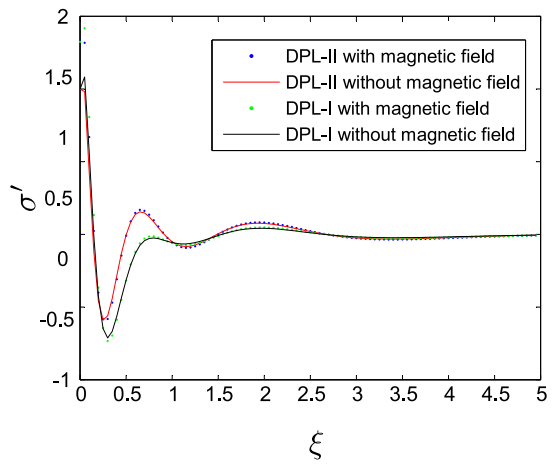

Fig. 8. Variation of stress $\sigma^{\prime}$ with distance time $=0.69$

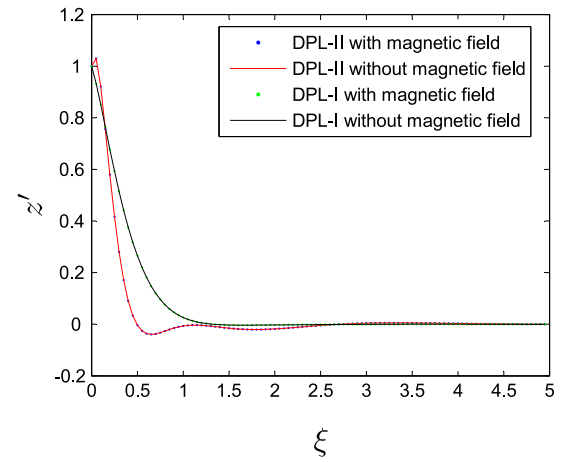

Fig. 3. Variation of temperature $z^{\prime}$ with distance $\xi$, time $=0.13$

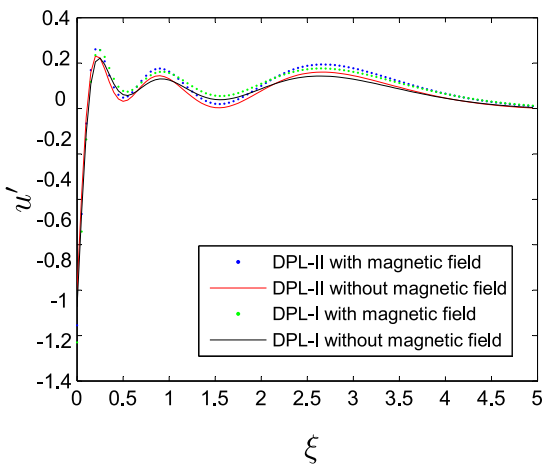

Fig. 6. Variation of displacement $u^{\prime}$ with distance $\xi$, time $=0.13$

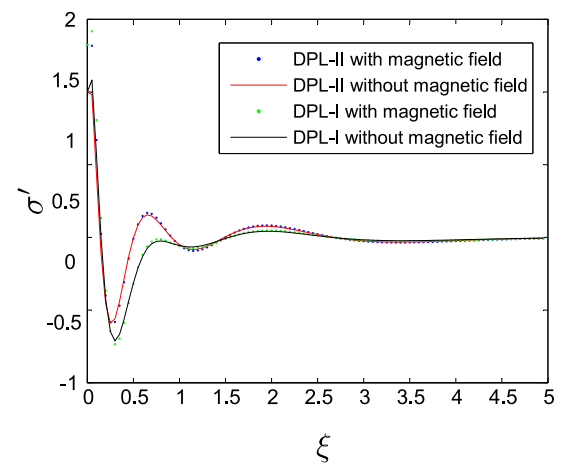

Fig. 9. Variation of stress $\sigma^{\prime}$ with distance $\xi$, time $=0.13$ but we observe that the non-dimensional temperature field achieves a negative value before approaching to zero value in MTDPL-II but it is always positive in MTDPL-I case.

BEHAVIOUR OF DISPLACEMENT UNDER MTDPLI AND MTDPL-II

Figs. 4, 5 and 6 represent the nature of displacement field at various times $\tau=1.21$ and 0.69 and 0.13 for MTDPL-I and MTDPL-II models. Here, the effect of magnetic field is prominent in both cases (MTDPL-I and MTDPL-II) at all three times, although its effect gradually decreases as the distance from boundary increases. The consequence of the presence of magnetic field is in such a manner that the value of displacement becomes lower in the presence of magnetic field at higher time but this behaviour becomes opposite at smaller time (i.e., at $\tau=0.69$ and $\tau=0.13$ ). Here the value 


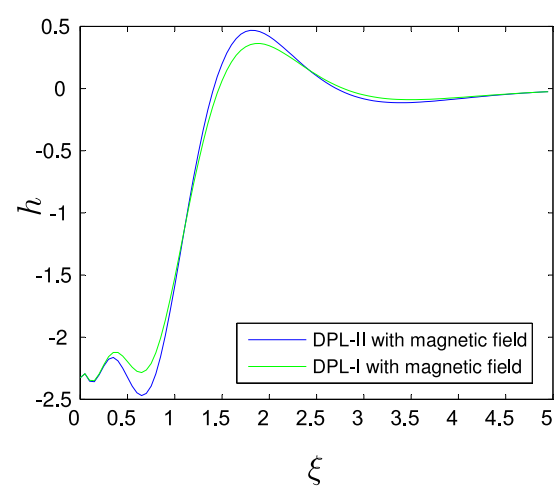

Fig. 10. Variation of perturbed magnetic field $h$ with distance $\xi$, time $=1.21$

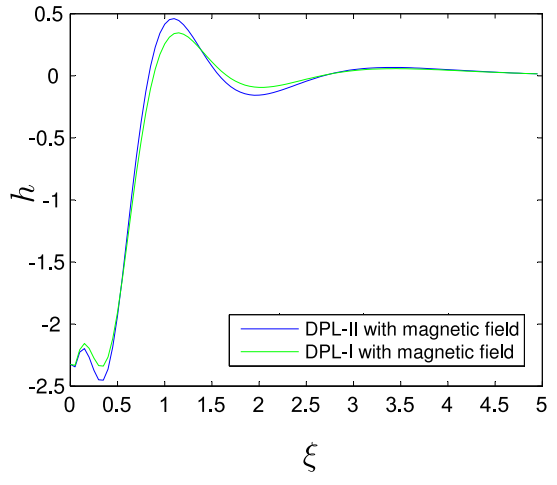

Fig. 11. Variation of perturbed magnetic field $h$ with distance $\xi$, time $=0.69$

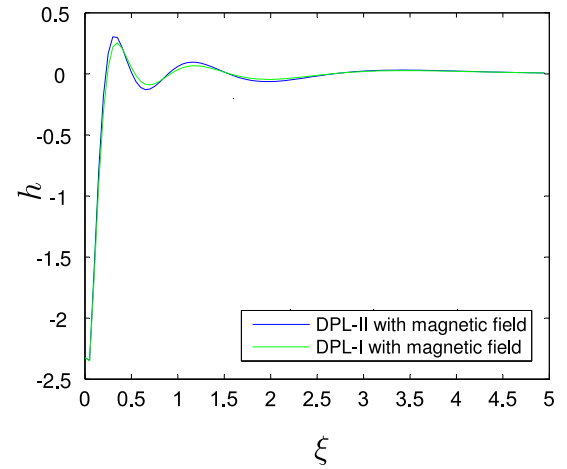

Fig. 12. Variation of perturbed magnetic field $h$ with distance $\xi$, time $=0.13$ of displacement becomes greater when we consider the presence of magnetic field in the medium. This fact is evident for both theories MTDPL-I and MTDPL-II. Furthermore, the maximum numerical value of displacement is greater in the MTDPL-II model in comparison to the results under MTDPL-I model and there is a prominent difference in predictions by MTDPL-I and MTDPL-II models.

\section{BEHAVIOUR OF STRESS UNDER MTDPL-I AND MTDPL-II}

Figs. 7, 8 and 9 exhibit the nature of stress field at different times 1.21 and 0.69 and 0.13 predicted by two models. It is noted that the influence of magnetic field is significant on the nature of stress field in the contexts of both the models. In the absence of magnetic field, when the distance $\xi=0$, the value of stress is 1 at all three times in cases of MTDPL-I and MTDPL-II models, but in the presence of magnetic field the value of stress becomes greater than 1 under both the theories. Furthermore, we also observe that the minimum value of stress in case of MTDPL-I is greater than the minimum value of stress in case of MTDPL-II at higher times, i.e., at $\tau=1.21$ and $\tau=0.69$ (see Figs. 7 and 8). However, Fig. 9 indicates that at very small time, the minimum value of stress in case of MTDPL-I is less than the minimum value of stress under the MTDPL-II model. Furthermore, the difference between two models decreases as time decreases.

\section{BEHAVIOUR OF PERTURBED MAGNETIC FIELD UNDER MTDPL-I AND MTDPL-II}

Figs. 10, 11 and 12 display the nature of perturbed magnetic field at different times 1.21 and 0.69 and 0.13 for two models. Here the difference between the predictions by two models is significant. The minimum value of perturbed magnetic field in MTDPL-II is lower than the minimum value of perturbed magnetic field for MTDPL-I. However, the maximum value of perturbed magnetic field in MTDPL-II is greater than the maximum value of perturbed magnetic field for MTDPL-I.

\section{CONCLUSION}

In the present work we employed the dual phase-lag magneto-thermoelasticity theory and studied a problem of elastic half space with finite conductivity permeated by a uniform magnetic field. The boundary of the half space is subjected to a normal load and a thermal shock that originate magneto-thermoelastic waves inside the medium.

We have presented a thorough analysis of the effects of magnetic field on wave propagation, and to investigate the nature of distributions of different fields like temperature, displacement, stress and perturbed magnetic field in the media in contexts of two models of magneto-thermoelasticity with dual phase-lags, namely MTDPL-I model and MTDPLII model. The numerical values of distributions of the physical fields for a suitable material have also been computed and displayed in graphical forms.

Significant differences among the analytical results predicted by two models MTDPL-I and MTDPL-II are observed. In the case of MTDPL-I we found that a solution of each field consists of two parts. The first one is a wave part that is identified as a modified elastic wave and the second part is not wave type, but of diffusive type. The nondimensional speed of elastic wave is found to be finite and equal to 1, i.e. the dimensionless speed of elastic wave is not effected by any of the phase-lag parameters $\tau_{q}^{\prime}$ and $\tau_{t}^{\prime}$ and it is also independent of the effect of the magnetic field. But the attenuation coefficient of modified elastic wave is dependent on phase-lag parameters $\tau_{q}^{\prime}$ and $\tau_{t}^{\prime}$. However, we note that the solution of each field variable in case of the MTDPL-II model consists of two coupled waves: modified elastic and modified thermal wave. The non-dimensional speed of both the waves is finite and dependent on phase-lag parameters 
$\tau_{q}^{\prime}$ and $\tau_{t}^{\prime}$ and magnetic field, too. Furthermore, we observe that temperature, stress and perturbed magnetic fields have discontinuities with finite jumps at both the elastic and thermal wave fronts and displacement is observed to be continuous in nature at both the wavefronts in the context of the MTDPL-II model. However, we obtain different results under the MTDPL-I model. We observe here that in this case, only stress and perturbed magnetic field show discontinuities having finite jumps at elastic wave front but the temperature and displacement are free from any discontinuities.

We also observe significant differences in the numerical results predicted by two different models. It is noted that the non dimensional temperature achieves a negative value for a region before approaching to zero value in MTDPL-II but it is always positive under MTDPL-I case. While observing the nature of stress, it is found that the minimum value of stress in MTDPL-I is greater than the minimum value of stress in MTDPL-II at higher time, although during initial time of interaction the minimum value of stress in MTDPLI is lower than the minimum value of stress in MTDPL-II. Furthermore, the maximum numerical value of displacement is greater in case of MTDPL-II as compared to the case of MTDPL-I.

The magnetic field is not prominently effective in the distribution of temperature in the contexts of both the MTDPL-I and MTDPL-II models, however, the stress field and displacement field are effected by the presence of magnetic field under both models.

\section{References}

[1] H.S. Carslaw, J.C. Jaeger, Conduction of Heat in Solids, Oxford University Press, London 1959.

[2] A.N. Tikhonov, A.A. Samarskii, Equations of Mathematical Physics, Dover New York 1990.

[3] J.I. Frankel, B. Vick, M.N. Ozisik, General formulation and analysis of hyperbolic heat conduction in composite media, Int. J. Heat Mass Transfer 30(7), 1293-1305 (1987).

[4] D.D. Joseph, L. Preziosi, Heat waves, Rev. Mod. Phys. 61(1), 41-73 (1989).

[5] L. Wang, X. Zhou, X. Wei, Heat Conduction: Mathematical Models and Analytical Solutions, Springer-Verlag, Berlin Heidelberg 2008.

[6] M.A. Biot, Thermoelasticity and irreversible thermodynamics, J. Appl. Mech. Tech. Phys. 27, 240-253 (1956).

[7] C. Cattaneo, A form of heat conduction equation which eliminates the paradox of instantaneous propagation, Compt. Rend. 247, 431-433 (1948).

[8] P. Vernotte, Some possible complications in the phenomenon of thermal conduction, Compt. Rend. 252, 2190-2191 (1961).

[9] H.W. Lord, Y. Shulman, A Generalized dynamical theory of thermoelasticity, J. Mech. Phys. Solids 15, 299-309 (1967).

[10] A.E. Green, K.A. Lindsay, Thermoelasticity, J. Elasticity 2 , 1-7 (1972).

[11] A.E. Green, P.M. Naghdi, Thermoelasticity without energy dissipation, J. Elasticity 31, 189-208 (1993).
[12] A.E. Green, P.M. Naghdi, A re-examination of the basic postulates of thermoemechanics, Proc. Roy. Soc. London A432, 171-194 (1991).

[13] D.Y. Tzou, Macro-to Microscale Heat Transfer: The Lagging Behavior, Taylor \& Francis, New York 1997.

[14] D.Y. Tzou, On the thermal shock wave induced by a moving heat source, ASME J. Heat Transfer 111(2), 232-238 (1989).

[15] J.R. Ho, C.P. Kuo, W.S. Jiaung, Study of heat transfer in multilayered structure within the framework of dual-phaselag heat conduction model using lattice Boltzmann method, Int. J. Heat Mass Transfer 46(1), 55-69 (2003).

[16] K. Ramadan, Semi-analytical Solutions for the dual phaselag heat conduction in multilayered media, Int. J. Therm. Sci. 48(1), 14-25 (2009).

[17] N.S. Al-Huniti, M.A. Al-Nimr, Thermoelastic behavior of a composite slab under a rapid dual-phase-lag heating, J. Therm. Stresses 27(7), 607-623 (2004).

[18] Y.M. Lee, T.W. Tsai, Ultra-fast pulse laser heating on a twolayered semi-infinite material with interfacial contact conductance, Int. Commun. Heat Mass Transfer 34(1), 45-51 (2007).

[19] K.C. Liu, Numerical analysis of dual-phase-lag heat transfer in a layered cylinder with nonlinear interface boundary conditions, Comput. Phys. Commun. 177(3), 307-314 (2007).

[20] D.S. Chandrasekharaiah, Hyperbolic thermoelasticity: a review of recent literature, Appl. Mech. Rev. 51(12), 705-729 (1998).

[21] W.S. Kim, L.G. Hector, M.N. Ozisik, Hyperbolic heat conduction due to axisymmetric continuous or pulsed surface heat sources, J. Applied Phys. 68, 5478-5485 (1990).

[22] M.N. Ozisik, D.Y. Tzou, On the wave theory in heat conduction, ASME Journal of Heat Transfer 116, 526-535 (1994).

[23] M.A. Al-Nimr, M. Naji, The Hyperbolic heat conduction equation in anisotropic material, Int. J. Thermophysics 21(1), 281-287 (2000).

[24] M.A. Al-Nimr, M. Naji, On the phase-lag effect on the non equilibrium entropy production, Microscale Thermophysical Engineering 4, 231-243 (2000).

[25] M.A. Al-Nimr, M. Naji,, V. Arpaci, Non equilibrium entropy production under the effect of dual-phase-lag heat conduction model, ASME J. Heat Transfer 122, 217-223 (2000).

[26] M.A. Al-Nimr, M. Hader, Melting and solidification under the effect of phase-lag concept in the hyperbolic conduction equation, Heat Transfer Engineering 22(2), 1-8 (2001).

[27] M.A. Al-Nimr, O.M. Haddad, The Phase-lag concept in the thermal behavior of lumped systems, Heat and Mass Transfer 37(2-3), 175-181 (2001).

[28] M.A. Al-Nimr, N. Al-Huniti, Transient thermal stresses in a thin elastic plate due to a rapid dual-phase-lag heating, J. Therm. Stresses 23, 731-746 (2000).

[29] J.L. Nowinski, Theory of Thermoelasticity with Applications, Sijthoff \& Noordhoff, International Alphen Aan Den Rijn 1978.

[30] N.F. Jordan, A.C. Eringen, On the static non linear theory of electromagnetic thermoelastic solids Part I, Int. J. Eng. Sci. 2, 59-95 (1964).

[31] N.F. Jordan, A.C. Eringen, On the static non linear theory of electromagnetic thermoelastic solids Part II, Int. J. Eng. Sci. 2, 97-114 (1964).

[32] G.A. Maugin, The mechanical behaviour of electromagnetic solids, Phil. Trans. Roy. Soc. 302,189-215 (1984).

[33] E. Radzikowska, R. Kotowski, W. Muschik, A non equilibrium evolution criterion for electromagnetic bodies, J. Non Equilibrium Thermodynamics 26(31), 215-230 (2001). 
[34] B.T. Maruszewski, A. Drzewiecki, R. Starosta, Anomalous features of the thermomagnetoelastic solid in a vortex array in a superconductor: Propagation of Love's waves, J. Therm. Stresses 30(9-10), 1049-1065 (2007).

[35] I-Shih Liu, Constitutive theory of anisotropic rigid heat conductors, Journal of Mathematical Physics 50(8), DOI: 10.1063/1.3190487 (2009).

[36] J. Casas-Vazquez, M. Criado-Sancho, D. Jou, Extended thermodynamics of polymers and superfluids, Journal of Nonnewtonian Fluid Mechanics 152(1-3), 36-44 (2008).

[37] G. Lebon, D. Jou, Early history of extended irreversible thermodynamics (1953-1983): An exploration beyond local equilibrium and classical transport theory, European Physical Journal H 40(2), 205-240 (2015).

[38] D. Jou, J. Casas-Vazquez, Extended irreversible thermodynamics and its relation with other continuum approaches, Journal of Non Newtonian Fluid Mechanics 96(1-2), 77-104 (2001).

[39] B.T. Maruszewski, A. Drzewiecki, R. Starosta, Thermodynamics of unconventional thermoelastic damping in auxetic media, Phys. Status Solidi B 250(10), 2044-2050 (2013).

[40] S. Kaliski, W. Nowacki, Excitation of mechanicalelectromagnetic waves induced by a thermal shock, BUll. Acad. Polon. Sci. Series. Sci. Tech. 1(10), (1962).

[41] C. Massalas, A. Dalamangas, Coupled magnetothermoelastic problem in elastic half-space, Lett. Appl. Eng. Sci. 21(8) (1983).
[42] S.K. Roychoudhuri, G. Chatterjee, A coupled magnetothermoelastic problem in a perfectly conducting half-space with thermal relaxation, Int. J. Math. and Math. Sci. 13(3), (1990).

[43] S.K. Roychoudhuri, G. Chatterjee, Temperature-rate dependent magneto-thermoelastic waves in a finitely conducting elastic half-space, Computers Math. Applic. 19(5), (1990).

[44] J.N. Sharma, S. Dayal Chand, Transient generalized magneto-thermoelastic waves in a half space, Int. J. Eng. Sci. 26 (9), (1988).

[45] S.K. Roychoudhuri, S. Banerjee (Mukhopadhyay), Magneto-Thermoelastic waves induced by a thermal shock in a finitely conducting elastic half space, Int. J. Math., Math. Sci. 19(1), 131-144 (1996).

[46] J.C. Maxwell, A dynamical theory of the electromagnetic field, Phil. Trans. Roy. Soc. 155, 459-512 (1865).

[47] R. Bellman, R.E. Kolaba, J.A. Lockette, Numerical Inversion of the Laplace Transform, American Elsevier Pub. Co., New York 1966.
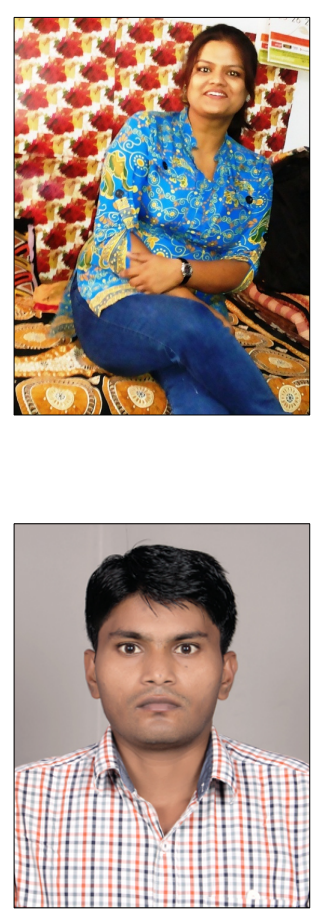

\section{Rakhi Tiwari,}

17 August, MSc in Mathematics, Banaras Hindu University, India, 2011; nationality: Indian; E-mail: rakhibhu2117@gmail.com, rakhitiwari.rs.apm12@itbhu.ac.in; Current Affiliation: Ph.D. Student at the Indian Institute of Technology (BHU), India; professional: mathematical modeling, theory of thermoelasticity; research work: 2 papers published, 3 conference presentations.

Mr. Anil Kumar, MSc in Mathematics, Indian Institute of Technology, New Delhi, India, 2012. E-mail: anilkumar12891@gmail.com, current affiliation: PhD Student, Indian Institute of Technology (BHU), India. 


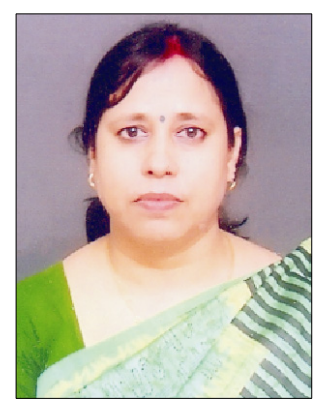

Santwana Mukhopadhyay, 7 January 1965, PhD in applied mathematics, University of Burdwan, India, 1998; nationality: Indian; E-mail: mukhosant.apm@itbhu.ac.in; membership: Mathematical Society, BHU; current affiliation: Associate Professor, Indian Institute of Technology (BHU), India; Professional: theory of thermoelasticity, computational mathematics; publications: nearly 70 papers in peer reviewed reputed journals and 10 conference presentations. 